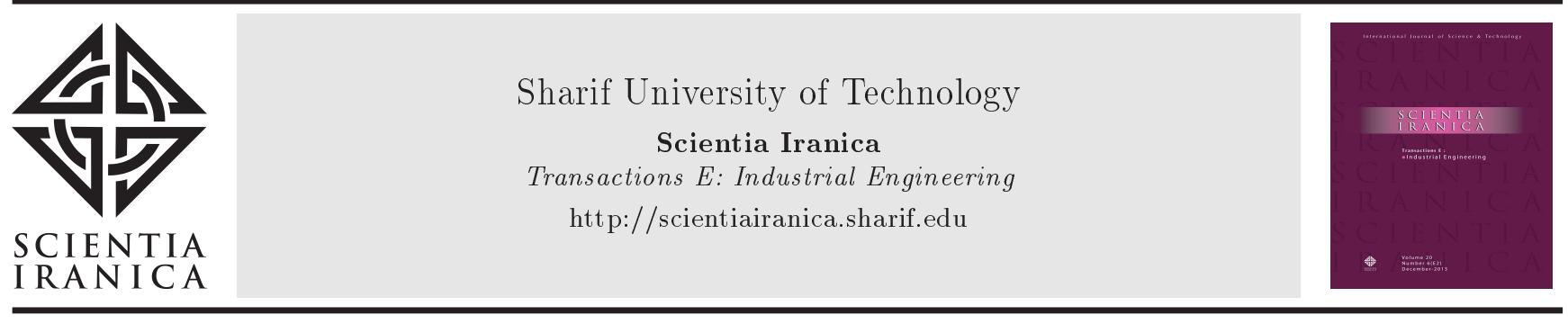

\title{
The algebraic structures of complex intuitionistic fuzzy soft sets associated with groups and subgroups
}

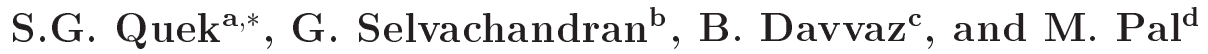 \\ a. A-Level Academy, UCSI College KL Campus, Lot 12734, Jalan Choo Lip Kung Taman Taynton View, 56000 Cheras, Kuala \\ Lumpur, Malaysia. \\ b. Department of Actuarial Science and Applied Statistics, Faculty of Business and Information Science, UCSI University, Jalan \\ Menara Gading, 56000 Cheras, Kuala Lumpur, Malaysia. \\ c. Department of Mathematics, Yazd University, Yazd, Iran. \\ d. Department of Applied Mathematics with Oceanology and Computer Programming, Vidyasagar University, West Bengal, India.
}

Received 19 December 2017; received in revised form 27 February 2018; accepted 23 April 2018

\author{
KEYWORDS \\ Fuzzy group; \\ Intuitionistic fuzzy \\ group; \\ Complex fuzzy set; \\ Complex intuitionistic \\ fuzzy soft set; \\ Fuzzy soft group.
}

\begin{abstract}
In recent years, the theory of complex fuzzy sets has captured the attention of many researchers, and research in this area has intensified over the past five years. This paper focuses on developing the algebraic structures pertaining to groups and subgroups for the complex intuitionistic fuzzy soft set model. Besides examining some of the properties of these structures, the relationship between these structures and corresponding structures in fuzzy group theory is also examined.
\end{abstract}

(C) 2019 Sharif University of Technology. All rights reserved.

\section{Introduction}

Uncertainty, imprecision, and vagueness are characteristics that are pervasive in problems occurring in the real world, and these features cannot be handled effectively using mathematical tools that are traditionally used to deal with uncertainties and vagueness. Some of the pioneering theories used to deal with these limitations include fuzzy set theory [1], intuitionistic fuzzy set theory [2], and soft set theory [3]. To overcome the problems that are inherent in each of these theories, researchers have chosen to combine these theories to develop new fuzzy-based hybrid models. The more well known among these include fuzzy soft sets [4],

*. Corresponding author.

E-mail addresses: queksg@ucsicollege.edu.my (S.G. Quek); Ganeshsree@ucsiuniversity.edu.my (G. Selvachandran); davvaz@yazd.ac.ir (B. Davvaz); mmpalvu@gmail.com (M. Pal)

doi: $10.24200 /$ sci.2018.50050.1485 intuitionistic fuzzy soft sets [5], interval-valued fuzzy soft sets [6], interval-valued intuitionistic fuzzy soft sets [7], and vague soft sets [8]. Although all of the above-mentioned theories are able to handle the uncertainties and fuzziness that exist in the data, all of these models are not able to handle the periodicity or seasonality that exists in many real-life problems. This led to the introduction of the complex fuzzy set model in [9] and, subsequently, the development and extension of this theory.

The notion of complex sets stems from the concept of complex numbers, which is a primary concept for solving problems, especially in the field of engineering. Complex sets notion, in practice, has the ability to solve many problems that cannot be solved using traditional mathematical concepts such as number theory, probability theory, and fuzzy set theory. Examples of these instances include solving the improper integrals that are used to represent resistance in electrical engineering and also represent the phase or wave-like qualities in two-dimensional problems. This 
led to the notion of complex fuzzy sets in [9], which is an improved and extended version of ordinary fuzzy sets. Kumar \& Bajaj [10], then, proposed the notion of Complex Intuitionistic Fuzzy Soft Sets (CIFSS) that combines the characteristics and advantages of complex sets, soft sets, and intuitionistic fuzzy sets in a single set. The CIFSS is parametric in nature and characterized by an amplitude term, which is equivalent to the membership and non-membership functions in an ordinary IFSS, and a phase term that represents the seasonality and/or periodicity of the elements. The novelty of CIFSS is manifested in the additional dimension of membership, which is the phase of the grade of membership. This feature gives CIFSS the added advantage of being able to represent data or information occurring repeatedly over a period of time, which is often the case with problems that are two-dimensional in nature.

Although research studies pertaining to the theory of CFSs and other complex fuzzy-based models are still in their infancy, they have been steadily gaining momentum in recent years. As of now, almost all of the work done in this area has revolved around the study of the theoretical properties of CFSs, complex fuzzy computing and modeling, complex fuzzy logic, complex fuzzy optimization and decision-making, and the application of these in solving time-periodic problems. The phase term in the structure of CFSs is the key defining feature of this model and can be used to model the seasonality and/or periodicity of timeperiodic phenomena. However, this is not the only interpretation for the phase term. Instead, the phase term can be used to represent different aspects of the information, depending on the context of the scope of the problem or area that is being studied. In most of the existing literature, the phase term has been used to represent the time factor and seasonality of the problems and has been applied to multi-attribute decision-making problems in a myriad of areas including supplier selection, economics, pattern recognition, engineering, and artificial intelligence.

The phase term can also be used to accurately represent the cycles present in fuzzy algebraic structures. In the study of complex fuzzy algebraic theory, the fuzzy algebraic structures are defined in a complex fuzzy setting; therefore, the structures consist of an amplitude term and a phase term. The amplitude term is equivalent to the membership function in ordinary fuzzy sets, whereas the phase term can be used to aptly represent the cycles of the algebraic structures. For example, when dealing with fuzzy alternating groups, different cycles can be represented aptly and accurately using the phase term if the fuzzy alternating groups are defined in terms of CFSs or any complex fuzzy-based models. This would make it easier to identify different cycles and their corresponding membership functions in a systematic manner. The desire to utilize this unique ability of the phase term present in the CFS model and other complex fuzzy-based models in the study of fuzzy algebra served as the main motivation to introduce and develop the theory of complex intuitionistic fuzzy soft groups in this paper. In this regard, the notion of CIFS groups and other supporting algebraic structures for CIFSGs are introduced and developed. The lack of proper research pertaining to the algebraic theory of complex fuzzy-based models in the literature served as another motivation for the study done in this paper.

The rest of this paper is organized as follows. In Section 2, some important background information pertaining to the concepts introduced here is recapitulated. In Section 3, the algebraic structures of complex intuitionistic fuzzy subgroups and complex intuitionistic fuzzy soft groups are derived, and the properties and structural characteristics of these algebraic structures are proposed and, subsequently, verified. The relationship between the structures introduced here and corresponding concepts in fuzzy group theory and classical group theory are also examined and verified in this section. In Section 4, normal complex intuitionistic fuzzy soft groups are proposed, and the properties of this structure are discussed and verified. Concluding remarks are presented in Section 5, followed by acknowledgments and a list of references.

\section{Preliminaries}

In this section, we recapitulate some of the important background information pertaining to the development of the algebraic structures that will be proposed here.

\subsection{Intuitionistic fuzzy sets}

An Intuitionistic Fuzzy Set (IFS) [2] is an extension of the classical fuzzy set and is characterized by a membership function and a non-membership function, each of which describes the degree of belongingness and non-belongingness of the elements with respect to each attribute. The concept of IFS was then further extended by incorporating the concept of soft set to derive the concept of Intuitionistic Fuzzy Soft Set (IFSS) [5].

In all that follows, $U$ shall be used to denote a universal set.

Definition 2.1 [2]. Let $A=\left\{\left(x, \mu_{A}(x), \nu_{A}(x)\right): x \in\right.$ $U\}$, where both $\mu_{A}$ and $\nu_{A}$ are functions from $U$ to $[0,1]$, satisfying $0 \leqslant \mu_{A}(x)+\nu_{A}(x) \leqslant 1$ for all $x \in U$. Then, $A$ is called an intuitionistic fuzzy set on $U$, where $\mu_{A}$ is the membership function of $A$ and $\nu_{A}$ is the nonmembership function of $A$.

Define $\pi_{A}(x)=1-\mu_{A}-\nu_{A}$. Then, for each $x_{0} \in U$ :

(i) The value of $\mu_{A}\left(x_{0}\right)$ is called the degree of belongingness of $x_{0}$ to $A$; 
(ii) The value of $\nu_{A}\left(x_{0}\right)$ is called the degree of nonbelongingness of $x_{0}$ to $A$;

(iii) The value of $\pi_{A}\left(x_{0}\right)$ is called the degree of uncertainty or indeterminacy of $x_{0}$ to $A$.

Henceforth, $A$ and $B$ shall be used to denote two intuitionistic fuzzy sets on $U$, which are defined below:

$$
\begin{aligned}
& A=\left\{\left(x, \mu_{A}(x), \nu_{A}(x)\right): x \in U\right\}, \\
& B=\left\{\left(x, \mu_{B}(x), \nu_{B}(x)\right): x \in U\right\} .
\end{aligned}
$$

Definition 2.2 [2]. The subset and equality of $A$ and $B$ are defined below:

(a) $A \subseteq B$, if $\mu_{A}(x) \leqslant \mu_{B}(x)$ and $\nu_{A}(x) \geqslant \nu_{B}(x)$ for all $x \in U$;

(b) $A=B$, if $A \subseteq B$ and $B \subseteq A$.

Definition 2.3 [2]. The complement, union, and intersection of $A$ and $B$ are defined below:

(a) $A=\left\{\left(x, \nu_{A}(x), \mu_{A}(x)\right): x \in U\right\}$;

(b) $A \cup B=\left\{\left(x, \max \left\{\mu_{A}(x), \mu_{B}(x)\right\}, \min \left\{\nu_{A}(x)\right.\right.\right.$, $\left.\left.\left.\nu_{B}(x)\right\}\right): x \in U\right\}$

(c) $A \cap B=\left\{\left(x, \min \left\{\mu_{A}(x), \mu_{B}(x)\right\}, \max \left\{\nu_{A}(x)\right.\right.\right.$, $\left.\left.\left.\nu_{B}(x)\right\}\right): x \in U\right\}$.

Definition 2.4 [2]. The set $\left\{x \in U: \nu_{A}(x)>0\right\}$ is called the support of $A$ and is denoted by $\mathscr{S}_{A}$. Moreover:

(a) $A$ is said to be null if $\mathscr{S}_{A}=\emptyset$; otherwise, it is said to be non-null;

(b) $A$ is said to be absolute if $\mathscr{S}_{A}=U$.

2.2. Soft sets and intuitionistic fuzzy soft sets Definition 2.5 [3]. Let $E$ be a set of parameters. Denote $\wp(U)$ to be the power set of $U$, and let $\mathcal{F}$ be a function from $E$ to $\wp(U)$. Then, the set of ordered pairs $\{(\varepsilon, \mathcal{F}(\varepsilon)): \varepsilon \in E, \mathcal{F}(\varepsilon) \in \wp(U)\}$, denoted by $(\mathcal{F}, E)$, is called a soft set on $U$. Moreover, for each $\varepsilon_{0} \in E$, $\mathcal{F}\left(\varepsilon_{0}\right)$ is called the set of $\varepsilon_{0}$-elements of $(\mathcal{F}, E)$, or the $\varepsilon_{0}$-approximate elements of $(\mathcal{F}, E)$.

Definition 2.6 [11]. Let $E$ be a set of parameters. Let $(\mathcal{F}, E)$ be a soft set on $U$. Then, the set $\{\varepsilon \in$ $E: \mathcal{F}(\varepsilon) \neq \emptyset\}$, denoted by $\mathscr{S}(\mathcal{F}, E)$, is called the support of $(\mathcal{F}, E)$. Moreover, $(\mathcal{F}, E)$ is said to be null if $\mathscr{S}(\mathcal{F}, E)=\emptyset$; otherwise, it is said to be non-null.

Definition 2.7 [5]. Let $E$ be a set of parameters. $\operatorname{IFS}(U)$ denotes a collection of all intuitionistic fuzzy sets on $U$ and let $\mathcal{F}$ be a function from $E$ to $\operatorname{IFS}(U)$. Then, the set of ordered pairs $\{(\varepsilon, \mathcal{F}(\varepsilon)): \varepsilon \in E, \mathcal{F}(\varepsilon) \in$ $\operatorname{IFS}(U)\}$, denoted by $(\mathcal{F}, E)$, is called an intuitionistic fuzzy soft set on $U$.
Definition 2.8 [5]. Let $(\mathcal{F}, E)$ be an intuitionistic fuzzy soft set on $U$. Then, the set $\{\varepsilon \in E: \mathcal{F}(\varepsilon) \neq \emptyset\}$, denoted by $\mathscr{S}(\mathcal{F}, E)$, is called the support of $(\mathcal{F}, E)$. Moreover, $(\mathcal{F}, E)$ is said to be null if $\mathscr{S}(\mathcal{F}, E)=\emptyset$; otherwise, it is said to be non-null.

Definition 2.9 [5]. Let $\left(\mathcal{F}_{1}, E_{1}\right)$ and $\left(\mathcal{F}_{2}, E_{2}\right)$ be two intuitionistic fuzzy soft sets on $U$. Then, $\left(\mathcal{F}_{1}, E_{1}\right)$ is an intuitionistic fuzzy soft subset of $\left(\mathcal{F}_{2}, E_{2}\right)$, denoted by $\left(\mathcal{F}_{1}, E_{1}\right) \widetilde{\widetilde{\subseteq}}\left(\mathcal{F}_{2}, E_{2}\right)$, if:

(i) $E_{1} \subseteq E_{2}$;

(ii) $\mathcal{F}_{1}(\varepsilon) \subseteq \mathcal{F}_{2}(\varepsilon)$ for all $\varepsilon \in \mathscr{S}\left(\mathcal{F}_{1}, E_{1}\right)$.

Remark. For each $\varepsilon \in \mathscr{S}\left(\mathcal{F}_{1}, E_{1}\right), \mathcal{F}_{1}(\varepsilon)$ is non-null. Thus, if $\left(\mathcal{F}_{1}, E_{1}\right) \widetilde{\subseteq}\left(\mathcal{F}_{2}, E_{2}\right)$, then $\mathcal{F}_{1}(\varepsilon) \subseteq \mathcal{F}_{2}(\varepsilon)$, and we also have $\mathcal{F}_{2}(\varepsilon)$ being non-null, which implies $\varepsilon \in$ $\mathscr{S}\left(\mathcal{F}_{2}, E_{2}\right)$. As a result, the condition $\mathscr{S}\left(\mathcal{F}_{1}, E_{1}\right) \subseteq$ $\mathscr{S}\left(\mathcal{F}_{2}, E_{2}\right)$ follows.

Definition 2.10 [5]. Let $\left(\mathcal{F}_{1}, E_{1}\right)$ and $\left(\mathcal{F}_{2}, E_{2}\right)$ be two intuitionistic fuzzy soft sets on $U$. Define $R=$ $E_{1} \cup E_{2}, S=E_{1} \cap E_{2}$; for all $\varepsilon \in S, \overline{\mathcal{H}}(\varepsilon)=\mathcal{F}_{1}(\varepsilon) \cup \mathcal{F}_{2}(\varepsilon)$ and $\overline{\mathcal{K}}(\varepsilon)=\mathcal{F}_{1}(\varepsilon) \cap \mathcal{F}_{2}(\varepsilon)$.

$$
\mathcal{H}(\varepsilon)= \begin{cases}\mathcal{F}_{1}(\varepsilon), & \varepsilon \in E_{1}-S \\ \mathcal{F}_{2}(\varepsilon), & \varepsilon \in E_{2}-S \\ \mathcal{F}_{1}(\varepsilon) \cup \mathcal{F}_{2}(\varepsilon), & \varepsilon \in S\end{cases}
$$

and:

$$
\mathcal{K}(\varepsilon)= \begin{cases}\mathcal{F}_{1}(\varepsilon), & \varepsilon \in E_{1}-S \\ \mathcal{F}_{2}(\varepsilon), & \varepsilon \in E_{2}-S \\ \mathcal{F}_{1}(\varepsilon) \cap \mathcal{F}_{2}(\varepsilon), & \varepsilon \in S\end{cases}
$$

Then:

(i) $(\mathcal{H}, R)$ is called the union of $\left(\mathcal{F}_{1}, E_{1}\right)$ and $\left(\mathcal{F}_{2}, E_{2}\right)$ and is denoted by $(\mathcal{H}, R)=\left(\mathcal{F}_{1}, E_{1}\right) \widetilde{\cup}\left(\mathcal{F}_{2}, E_{2}\right)$;

(ii) $(\mathcal{K}, R)$ is called the intersection of $\left(\mathcal{F}_{1}, E_{1}\right)$ and $\left(\mathcal{F}_{2}, E_{2}\right)$ and is denoted by $(\mathcal{K}, R)=$ $\left(\mathcal{F}_{1}, E_{1}\right) \widetilde{\cap}\left(\mathcal{F}_{2}, E_{2}\right) ;$

(iii) $(\overline{\mathcal{H}}, S)$ is called the restricted union of $\left(\mathcal{F}_{1}, E_{1}\right)$ and $\left(\mathcal{F}_{2}, E_{2}\right)$ and is denoted by $(\overline{\mathcal{H}}, S)=$ $\left(\mathcal{F}_{1}, E_{1}\right) \widehat{\cup}\left(\mathcal{F}_{2}, E_{2}\right)$;

(iv) $(\overline{\mathcal{K}}, S)$ is called the restricted intersection of $\left(\mathcal{F}_{1}, E_{1}\right)$ and $\left(\mathcal{F}_{2}, E_{2}\right)$ and is denoted by $(\overline{\mathcal{K}}, S)=$ $\left(\mathcal{F}_{1}, E_{1}\right) \widehat{\cap}\left(\mathcal{F}_{2}, E_{2}\right)$;

\subsection{Complex fuzzy sets}

In this section, an overview of the concept of Complex Fuzzy Sets (CFS) [9] and Complex Intuitionistic Fuzzy Soft Sets (CIFSS) [10] is presented. Since the introduction of CFS, attempts to improve and overcome the drawbacks that are inherent in the CFS model have led to the introduction of several complex fuzzy-based hybrid models. We refer the readers to [10,12-18] for more details on these models. 
Definition 2.11 [9]. A complex fuzzy set $A$ defined on a universe of discourse $U$ is characterized by a membership function $\mu_{A}(x)$ that assigns a complexvalued grade of membership in $A$ toany element $x \in$ $U$. By definition, all values of $\mu_{A}(x)$ lie within the unit circle on the complex plane and are expressed by $\mu_{A}(x)=r_{A}(x) \mathrm{e}^{\mathrm{i} \omega_{A}(x)}$, where $\mathrm{i}=\sqrt{-1}, r_{A}(x)$ and $\omega_{A}(x)$ are both real-valued, $r_{A}(x) \in[0,1]$, and $\omega_{A}(x) \in(0,2 \pi]$. A complex fuzzy set $A$ is thus of the following form:

$$
\begin{aligned}
A & =\left\{\left(x, \mu_{A}(x)\right): x \in U\right\} \\
& =\left\{\left(x, r_{A}(x) \mathrm{e}^{\mathrm{i} \omega_{A}(x)}\right): x \in U\right\} .
\end{aligned}
$$

Henceforth, symbol $\mathrm{i}$ is used to denote the imaginary unit $\sqrt{-1}$, whereas symbol $\mathrm{O}_{1}$ is used to denote $\{z \in$ $\mathbb{C}:|z| \leqslant 1\}$. Up until Section 2.2 , we have reached the concept of Intuitionistic Fuzzy Soft Sets (IFSS), involving the relations $\geqslant, \leqslant, \max$, min on the outcomes of membership and non-membership functions of the IFSS model. Such relations are inherently defined for real numbers only. On the other hand, a CFS and all its generalizations have membership and non-membership functions that can lie anywhere in $\mathrm{O}_{1}$. We must, therefore, generalize the concept of $\geqslant, \leqslant, \max , \min$ for all complex numbers in $\mathrm{O}_{1}$. To achieve these, the following definitions and lemmas are given.

Definition 2.12. Let $\mu=r \mathrm{e}^{\mathrm{i} \omega}$ and $\nu=\tau \mathrm{e}^{\mathrm{i} \psi}$, with $r, \tau \in[0,1]$ and $\omega, \psi \in(0,2 \pi]$. The relations $\geqslant$ and $\leqslant$ are given as follows:

(i) $\mu \geqslant \nu$, when both $r \geqslant \tau$ and $\omega \geqslant \psi$, or when $\nu=0$;

(ii) $\mu \leqslant \nu$, when both $r \leqslant \tau$ and $\omega \leqslant \psi$, or when $\mu=0$.

Remark. The usual definition of $\geqslant$ and $\leqslant$ at the real interval $[0,1]$ is a special case of this definition. However, there remain pairs of elements of $\mathrm{O}_{1}$ such that neither $\geqslant$ nor $\leqslant$ can be established between them, such as $0.1 \mathrm{e}^{3 \mathrm{i}}$ and $0.4 \mathrm{e}^{2 \mathrm{i}}$, because $0.1<0.4$, but $3>2$. Nonetheless, $0 \leqslant \mu \leqslant 1$ still holds for all $\mu \in \mathrm{O}_{1}$.

Definition 2.13. Let $S=\left\{\mu_{n}: n \in V\right\} \subseteq \mathrm{O}_{1}$. Then, $\max S$ and $\min S$ are as defined below:

(i) (a) $\max S \geqslant \mu_{n}$ for all $n \in V$;

(b) If $\xi \in \mathrm{O}_{1}$ is such that $\xi \geqslant \mu_{n}$ for all $n \in V$, then $\xi \geqslant \max S$;

(ii) (a) $\min S \leqslant \mu_{n}$ for all $n \in V$;

(b) If $\zeta \in \mathrm{O}_{1}$ is such that $\zeta \leqslant \mu_{n}$ for all $n \in V$, then $\zeta \leqslant \max S$.
Remark. Unlike subsets of $\mathbb{R}, \max S$ and $\min S$ may not be in $S$, even if $S$ is finite. For example, if $S_{0}=$ $\left\{0.1 \mathrm{e}^{3 \mathrm{i}}, 0.4 \mathrm{e}^{2 \mathrm{i}}\right\}$, then $\max S_{0}=0.4 \mathrm{e}^{3 \mathrm{i}}$ and $\min S_{0}=$ $0.1 \mathrm{e}^{2 \mathrm{i}}$.

Definition 2.14. Let $\mu=r \mathrm{e}^{\mathrm{i} \omega}$, with $r \in[0,1]$ and $\omega \in(0,2 \pi]$. The complement of $\mu$, denoted by $1 \sim \mu$, is defined as $1 \sim \mu=(1-r) \mathrm{e}^{\mathrm{i} \omega^{\prime}}$, where:

$$
\omega^{\prime}= \begin{cases}2 \pi-\omega, & \omega<2 \pi \\ \omega, & \omega=2 \pi\end{cases}
$$

Remark. If $\mu \in[0,1]$, then $1 \sim \mu=1-\mu$.

Lemma 2.1. For all $\mu \in O_{1}, 1 \sim(1 \sim \mu)=\mu$.

Remark. Let $\mu=r \mathrm{e}^{\mathrm{i} \omega}$, with $r \in[0,1]$ and $\omega \in$ $(0,2 \pi]$. Then, $|\mu|=r$.

Lemma 2.2. For all $\mu \in O_{1},|1 \sim \mu|=1-|\mu|$.

\subsection{Complex intuitionistic fuzzy soft sets}

The object of study in this paper is the CIFSS model [10], which is an adaptation of the original CFS model [9]. It is a hybrid composed of complex fuzzy sets, intuitionistic fuzzy sets, and soft sets characterized by membership and non-membership functions that represent the degree of belongingness and nonbelongingness of the elements with respect to the attributes that are under consideration.

Definition 2.15 [10]. Let $E$ be a set of parameters, $\operatorname{CIFS}(U)$ be the collection of all complex intuitionistic fuzzy sets on $U$, and $\widetilde{\mathcal{F}}$ be a function from $E$ to $\operatorname{CIFS}(U)$. Then, the set of ordered pairs $\{(\varepsilon, \widetilde{\mathcal{F}}(\varepsilon))$ : $\varepsilon \in E, \widetilde{\mathcal{F}}(\varepsilon) \in \operatorname{CIFS}(U)\}$, denoted by $(\widetilde{\mathcal{F}}, E)$, is called a Complex Intuitionistic Fuzzy Soft Set (CIFSS) on $U$. Note that, for each $\varepsilon \in E$ :

$$
\begin{aligned}
\widetilde{\mathcal{F}}(\varepsilon) & =\left\{\left(x, \mu_{\tilde{\mathcal{F}}(\varepsilon)}(x), \nu_{\widetilde{\mathcal{F}}(\varepsilon)}(x)\right): x \in U\right\} \\
= & \left\{\left(x, r_{\widetilde{\mathcal{F}}(\varepsilon)}(x) \mathrm{e}^{\mathrm{i} \omega_{\tilde{\mathcal{F}}(\varepsilon)}(x)}, \tau_{\widetilde{\mathcal{F}}(\varepsilon)}(x) \mathrm{e}^{\mathrm{i} \psi_{\tilde{\mathcal{F}}(\varepsilon)}(x)}\right): x \in U\right\} .
\end{aligned}
$$

In all that follows, let $\operatorname{CIFSS}(U)$ denote the collection of all complex intuitionistic fuzzy soft sets on a universe $U$. Furthermore, we write $(\mathcal{\mathcal { F }}, E) \in \operatorname{CIFSS}(U)$ to denote that $(\widetilde{\mathcal{F}}, E)$ is a complex intuitionistic fuzzy soft set on $U$.

Definition 2.16. Let $(\widetilde{\mathcal{F}}, E) \in \operatorname{CIFSS}(U)$. Then, the set $\{\varepsilon \in E: \widetilde{\mathcal{F}}(\varepsilon)$ is non-null $\}$, denoted by $\mathscr{S}(\widetilde{\mathcal{F}}, E)$, and is called the support of $(\widetilde{\mathcal{F}}, E)$. Moreover, $(\widetilde{\mathcal{F}}, E)$ is said to be null if $\mathscr{S}(\widetilde{\mathcal{F}}, E)=\emptyset$; otherwise, it is said to be non-null. 
Definition 2.17 [10]. Let $\left(\widetilde{\mathcal{F}}_{1}, E_{1}\right),\left(\widetilde{\mathcal{F}}_{2}, E_{2}\right) \quad \in$ $\operatorname{CIFSS}(U)$. Then, $\left(\widetilde{\mathcal{F}}_{1}, E_{1}\right)$ is a complex intuitionistic fuzzy soft subset of $\left(\widetilde{\mathcal{F}}_{2}, E_{2}\right)$, denoted as $\left(\widetilde{\mathcal{F}}_{1}, E_{1}\right) \widetilde{\subseteq}\left(\widetilde{\mathcal{F}}_{2}, E_{2}\right)$, if:

(i) $E_{1} \subseteq E_{2}$;

(ii) $\widetilde{\mathcal{F}}_{1}(\varepsilon) \subseteq \widetilde{\mathcal{F}}_{2}(\varepsilon)$ for all $\varepsilon \in \mathscr{S}\left(\widetilde{\mathcal{F}}_{1}, E_{1}\right)$.

Remark. When $\left(\widetilde{\mathcal{F}}_{1}, E_{1}\right) \widetilde{\subseteq}\left(\widetilde{\mathcal{F}}_{2}, E_{2}\right)$ for each $\varepsilon \in$ $\mathscr{S}\left(\widetilde{\mathcal{F}}_{1}, E_{1}\right), \widetilde{\mathcal{F}}_{1}(\varepsilon)$ is non-null. Since $\widetilde{\mathcal{F}}_{1}(\varepsilon) \subseteq \widetilde{\mathcal{F}}_{2}(\varepsilon)$, we also have $\widetilde{\mathcal{F}}_{2}(\varepsilon)$ being non-null, which implies $\varepsilon \in$ $\mathscr{S}\left(\widetilde{\mathcal{F}}_{2}, E_{2}\right)$. As a result, the condition $\mathscr{S}\left(\widetilde{\mathcal{F}}_{1}, E_{1}\right) \subseteq$ $\mathscr{S}\left(\widetilde{\mathcal{F}}_{2}, E_{2}\right)$ follows.

Definition 2.18 [10]. Let $(\widetilde{\mathcal{F}}, E) \in \operatorname{CIFSS}(U)$. Then, the complement of $(\widetilde{\mathcal{F}}, E)$, denoted by $(\widetilde{\mathcal{F}}, E)^{c}$, is defined as $(\widetilde{\mathcal{F}}, E)^{c}=\left(\widetilde{\mathcal{F}}^{c}, \neg E\right)$, where $\widetilde{\mathcal{F}}^{c}$ is a function from $\neg E$ to $\operatorname{CIFS}(U)$ given by:

$$
\begin{aligned}
\tilde{\mathcal{F}}^{c}(\neg \varepsilon) & =\left\{\left(x, \nu_{\widetilde{\mathcal{F}}(\neg \neg \varepsilon)}(x), \mu_{\widetilde{\mathcal{F}}(\neg \neg \varepsilon)}(x)\right): x \in U\right\} \\
& =\left\{\left(x, \nu_{\widetilde{\mathcal{F}}(\varepsilon)}(x), \mu_{\widetilde{\mathcal{F}}(\varepsilon)}(x)\right): x \in U\right\},
\end{aligned}
$$

for all $\neg \varepsilon \in \neg E$.

Remark. Definition 2.18 can be restated as follows. Let $(\widetilde{\mathcal{F}}, E) \in \operatorname{CIFSS}(U)$. Define $\widetilde{\mathcal{T}}$ as a function from $E$ to $\operatorname{CIFS}(U)$, where $\widetilde{\mathcal{T}}(\varepsilon)$ is the complement of $\widetilde{\mathcal{F}}(\varepsilon)$ for all $\varepsilon \in E$. Then, $(\widetilde{\mathcal{T}}, E)$ is called the complement of $(\tilde{\mathcal{F}}, E)$ and this can be denoted as $(\widetilde{\mathcal{T}}, E)=(\widetilde{\mathcal{F}}, E)^{c}$.

Note that, for each $\varepsilon \in E, \widetilde{\mathcal{T}}(\varepsilon)=\left\{\left(x, \nu_{\widetilde{\mathcal{F}}(\varepsilon)}\right.\right.$ $\left.\left.(x), \mu_{\tilde{\mathcal{F}}(\varepsilon)}(x)\right): x \in U\right\}$ in line with Definition 2.3.

Definition 2.19 [10]. Let $\left(\widetilde{\mathcal{F}}_{1}, E_{1}\right),\left(\widetilde{\mathcal{F}}_{2}, E_{2}\right) \quad \in$ $\operatorname{CIFSS}(U)$. Define $R=E_{1} \cup E_{2}, S=E_{1} \cap E_{2}$; and for all $\varepsilon \in S, \overline{\mathcal{H}}(\varepsilon)=\widetilde{\mathcal{F}}_{1}(\varepsilon) \cup \widetilde{\mathcal{F}}_{2}(\varepsilon)$ and $\overline{\mathcal{K}}(\varepsilon)=\widetilde{\mathcal{F}}_{1}(\varepsilon) \cap \widetilde{\mathcal{F}}_{2}(\varepsilon)$.

$$
\mathcal{H}(\varepsilon)= \begin{cases}\widetilde{\mathcal{F}}_{1}(\varepsilon), & \varepsilon \in E_{1}-S \\ \widetilde{\mathcal{F}}_{2}(\varepsilon), & \varepsilon \in E_{2}-S \\ \widetilde{\mathcal{F}}_{1}(\varepsilon) \cup \widetilde{\mathcal{F}}_{2}(\varepsilon), & \varepsilon \in S\end{cases}
$$

and:

$$
\mathcal{K}(\varepsilon)= \begin{cases}\widetilde{\mathcal{F}}_{1}(\varepsilon), & \varepsilon \in E_{1}-S \\ \widetilde{\mathcal{F}}_{2}(\varepsilon), & \varepsilon \in E_{2}-S \\ \widetilde{\mathcal{F}}_{1}(\varepsilon) \cap \widetilde{\mathcal{F}}_{2}(\varepsilon), & \varepsilon \in S\end{cases}
$$

Then:

(i) $(\mathcal{H}, R)$ is called the union of $\left(\widetilde{\mathcal{F}}_{1}, E_{1}\right)$ and $\left(\widetilde{\mathcal{F}}_{2}, E_{2}\right)$ and is denoted by $(\mathcal{H}, R)=\left(\widetilde{\mathcal{F}}_{1}, E_{1}\right) \widetilde{\cup}\left(\widetilde{\mathcal{F}}_{2}, E_{2}\right)$; (ii) $(\mathcal{K}, R)$ is called the intersection of $\left(\widetilde{\mathcal{F}}_{1}, E_{1}\right)$ and $\left(\widetilde{\mathcal{F}}_{2}, E_{2}\right)$ and is denoted by $(\mathcal{K}, R)=$ $\left(\widetilde{\mathcal{F}}_{1}, E_{1}\right) \widetilde{\cap}\left(\widetilde{\mathcal{F}}_{2}, E_{2}\right)$;

(iii) $(\overline{\mathcal{H}}, S)$ is called the restricted union of $\left(\widetilde{\mathcal{F}}_{1}, E_{1}\right)$ and $\left(\widetilde{\mathcal{F}}_{2}, E_{2}\right)$ and is denoted by $(\overline{\mathcal{H}}, S)=$ $\left(\widetilde{\mathcal{F}}_{1}, E_{1}\right) \widehat{\cup}\left(\widetilde{\mathcal{F}}_{2}, E_{2}\right)$;

(iv) $(\overline{\mathcal{K}}, S)$ is called the restricted intersection of $\left(\widetilde{\mathcal{F}}_{1}, E_{1}\right)$ and $\left(\widetilde{\mathcal{F}}_{2}, E_{2}\right)$ and is denoted by $(\overline{\mathcal{K}}, S)=$ $\left(\widetilde{\mathcal{F}}_{1}, E_{1}\right) \widehat{\cap}\left(\widetilde{\mathcal{F}}_{2}, E_{2}\right)$.

We now define two new operations for the CIFSS model, namely the $(\alpha, \beta)$-level set and the characteristic set of a CIFSS, and provide some properties of these operations. The formal definitions of these operations and the properties of these operations are given below.

Definition 2.20. Let $(\widetilde{\mathcal{F}}, E) \in \operatorname{CIFSS}(U)$, and $\alpha, \beta \in \mathrm{O}_{1}$. The $(\alpha, \beta)$-level set of $(\widetilde{\mathcal{F}}, E)$, denoted by $(\widetilde{\mathcal{F}}, E)_{(\alpha, \beta)}$, is a soft set on $U$ defined below:

$$
(\widetilde{\mathcal{F}}, E)_{(\alpha, \beta)}=\left\{\left(\varepsilon, \widetilde{\mathcal{F}}_{(\alpha, \beta)}(\varepsilon)\right): \varepsilon \in E, \widetilde{\mathcal{F}}_{(\alpha, \beta)}(\varepsilon) \in \wp(U)\right\},
$$

where $\widetilde{\mathcal{F}}_{(\alpha, \beta)}(\varepsilon)=\left\{x \in U: \mu_{\widetilde{\mathcal{F}}(\varepsilon)}(x) \geqslant \alpha, \nu_{\widetilde{\mathcal{F}}(\varepsilon)}(x) \leqslant\right.$ $\beta\}$ for all $\varepsilon \in E$.

If $\alpha=\beta$, then $(\widetilde{\mathcal{F}}, E)_{(\alpha, \alpha)}$ is called the $\alpha$-level set of $(\widetilde{\mathcal{F}}, E)$, denoted by $(\widetilde{\mathcal{F}}, E)_{\alpha}$, and defined as:

$$
(\widetilde{\mathcal{F}}, E)_{\alpha}=\left\{\left(\varepsilon, \widetilde{\mathcal{F}}_{\alpha}(\varepsilon)\right): \varepsilon \in E, \widetilde{\mathcal{F}}_{\alpha}(\varepsilon) \in \wp(U)\right\},
$$

where $\widetilde{\mathcal{F}}_{\alpha}(\varepsilon)=\left\{x \in U: \mu_{\widetilde{\mathcal{F}}(\varepsilon)}(x) \geqslant \alpha, \nu_{\widetilde{\mathcal{F}}(\varepsilon)}(x) \leqslant \alpha\right\}$ for all $\varepsilon \in E$.

Remark. Note that since $\widetilde{\mathcal{F}}_{(\alpha, \beta)}(\varepsilon) \in \wp(U)$ for all $\varepsilon \in E$, we have:

$$
(\widetilde{\mathcal{F}}, E)_{(\alpha, \beta)}=\left\{\left(\varepsilon, \widetilde{\mathcal{F}}_{(\alpha, \beta)}(\varepsilon)\right): \varepsilon \in E\right\} .
$$

Definition 2.21. Let $(\widetilde{\mathcal{F}}, E) \in \operatorname{CIFSS}(U)$ and $S$ be a non-null proper subset of $U$. If $\left\{\mu_{\widetilde{\mathcal{F}}(\varepsilon)}: \varepsilon \in E\right\}=\mu_{0}$ and $\left\{\nu_{\widetilde{\mathcal{F}}(\varepsilon)}: \varepsilon \in E\right\}=\nu_{0}$, in which:

$$
\mu_{0}(x)= \begin{cases}r \mathrm{e}^{\mathrm{i} \omega}, & x \in S \\ 1 \sim r \mathrm{e}^{\mathrm{i} \omega}, & x \in U-S\end{cases}
$$

and:

$$
\nu_{0}(x)= \begin{cases}\tau \mathrm{e}^{\mathrm{i} \psi}, & x \in S \\ 1 \sim \tau \mathrm{e}^{\mathrm{i} \psi}, & x \in U-S\end{cases}
$$

where $\omega+\psi \in\{2 \pi, 4 \pi\}$ and $r \mathrm{e}^{\mathrm{i} \omega} \geqslant \tau \mathrm{e}^{\mathrm{i} \psi}$, and then $(\widetilde{\mathcal{F}}, E)$ is said to be characteristic over $S$. 
Remark. Consider the particular case where $U=$ $\{p, q\}$ and $\mu_{0}(x)=\nu_{0}(x)=\frac{1}{2}$ for all $x \in U$.

Note that $r \mathrm{e}^{\mathrm{i} \omega}=\tau \mathrm{e}^{\mathrm{i} \psi}=r \mathrm{e}^{\mathrm{i} \omega}=\tau \mathrm{e}^{\mathrm{i} \psi}=\frac{1}{2} \in \mathrm{O}_{1}$, and $\omega+\psi=4 \pi, r=\tau$, and $\omega=\psi$. However, $S$ can be either $\{p\}$ or $\{q\}$. We now have an example of $(\widetilde{\mathcal{F}}, E)$ as characteristic over more than one non-null proper subset of $U$.

Proposition 2.1. Let $(\widetilde{\mathcal{F}}, E) \in \operatorname{CIFSS}(U)$, and $(\widetilde{\mathcal{F}}, E)$ be characteristic over $S$, in which:

$$
\mu_{0}(x)= \begin{cases}r \mathrm{e}^{\mathrm{i} \omega}, & x \in S \\ 1 \sim r \mathrm{e}^{\mathrm{i} \omega}, & x \in U-S\end{cases}
$$

and:

$$
\nu_{0}(x)= \begin{cases}\tau \mathrm{e}^{\mathrm{i} \psi}, & x \in S \\ 1 \sim \tau \mathrm{e}^{\mathrm{i} \psi}, & x \in U-S\end{cases}
$$

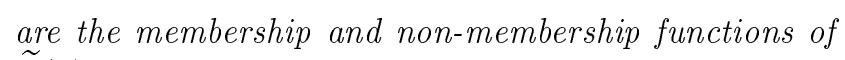
$\widetilde{\mathcal{F}}(\varepsilon)$, respectively. Then:

(i) $r+\tau=1$;

(ii) $r \mathrm{e}^{\mathrm{i} \omega} \geqslant 1 \sim r \mathrm{e}^{\mathrm{i} \omega}$ and $\tau \mathrm{e}^{\mathrm{i} \psi} \leqslant 1 \sim \tau \mathrm{e}^{\mathrm{i} \psi}$.

\section{Proof.}

(i) Note that $\mu_{0}$ and $\nu_{0}$ are the membership and the non-membership functions of $\widetilde{\mathcal{F}}(\varepsilon)$, which is a complex intuitionistic fuzzy set. Then, the condition $0 \leqslant\left|\mu_{0}(x)\right|+\left|\mu_{0}(x)\right| \leqslant 1$ holds for all $x \in U$. We now have both $0 \leqslant r+\tau \leqslant 1$ and $0 \leqslant\left|1 \sim r \mathrm{e}^{\mathrm{i} \omega}\right|+\left|1 \sim \tau \mathrm{e}^{\mathrm{i} \psi}\right| \leqslant 1$ by Definition 2.21 . From Lemma 2.2, it follows that:

$$
\begin{gathered}
\left|1 \sim r \mathrm{e}^{\mathrm{i} \omega}\right|+\left|1 \sim \tau \mathrm{e}^{\mathrm{i} \psi}\right|=(1-r)+(1-\tau) \\
=2-(r+\tau)
\end{gathered}
$$

causing $0 \leqslant 2-(r+\tau) \leqslant 1$; therefore, $1 \leqslant r+\tau \leqslant 2$, which implies $r+\tau=1$;

(ii) $\operatorname{As}(\widetilde{\mathcal{F}}, E)$ is characteristic over $S, \omega+\psi \in\{2 \pi, 4 \pi\}$ and $r \mathrm{e}^{\mathrm{i} \omega} \geqslant \tau \mathrm{e}^{\mathrm{i} \psi}$. Since $r \geqslant \tau$ and $r+\tau=1$, it follows that $r \geqslant \frac{1}{2}$ and $\tau \leqslant \frac{1}{2}$. Thus, we have $1-r \leqslant \frac{1}{2}$ and $1-\tau \geqslant \frac{1}{2}$. These further imply that $r \geqslant 1-r$ and $\tau \leqslant 1-\tau$.

Now, suppose that $\omega+\psi=2 \pi$. Since $\omega \geqslant \psi$, it follows that $\omega \geqslant \pi$ and $\psi \leqslant \pi$. We now have $1-\omega \leqslant \pi$ and $1-\psi \geqslant \pi$, implying that $\omega \geqslant 2 \pi-\omega$ and $\psi \leqslant$ $2 \pi-\psi$. In addition, note that both $\omega, \psi<2 \pi$. By Definition 2.14, we have $r \mathrm{e}^{\mathrm{i} \omega} \geqslant(1-r) \mathrm{e}^{\mathrm{i}(2 \pi-\omega)}=1 \sim$ $r \mathrm{e}^{\mathrm{i} \omega}$ and $\tau \mathrm{e}^{\mathrm{i} \psi} \leqslant(1-\tau) \mathrm{e}^{\mathrm{i}(2 \pi-\psi)}=1 \sim \tau \mathrm{e}^{\mathrm{i} \psi}$.

On the other hand, if $\omega+\psi=4 \pi$, then $\omega=$ $\psi=2 \pi$. Then, by Definition 2.14 , we have $r \mathrm{e}^{\mathrm{i} \omega} \geqslant$ $(1-r) \mathrm{e}^{\mathrm{i} \omega}=1 \sim r \mathrm{e}^{\mathrm{i} \omega}$ and $\tau \mathrm{e}^{\mathrm{i} \psi} \leqslant(1-\tau) \mathrm{e}^{\mathrm{i} \psi}=1 \sim \tau \mathrm{e}^{\mathrm{i} \psi}$.

\section{Complex intuitionistic fuzzy soft groups}

The study of soft algebra and fuzzy soft algebra was initiated by Aktas \& Cagman [19] and Aygunoglu \& Aygun [20], respectively. Other researchers such as Feng et al. [11], Acar et al. [21], Inan and Ozturk [22], and Ghosh et al. [23] also contributed to the development of these areas. Besides, many more advanced algebraic structures pertaining to groups, rings, and hemirings of fuzzy soft sets have been introduced in the literature. Some of the latest works include the introduction of soft fuzzy rough rings and ideals by Zhu [24], I-fuzzy soft groups by Vimala et al. [25], soft union set characterizations of hemirings by Zhan et al. [26], and neutrosophic normal soft groups by Bera and Mahapatra [27]. Yamak et al. [28], LeoreanuFotea et al. [29], and Selvachandran and Salleh [3034], on the other hand, were responsible for introducing the algebraic structures of soft hypergroupoids, fuzzy soft hypergroups as well as soft hyperrings, fuzzy soft hyperrings, vague soft hypergroups, and hyperrings, respectively. Khan et al. [35] proposed the notion of soft interior hyperideals of ordered semihypergroups, whereas $\mathrm{Ma}$ et al. [36] studied the concept of rough soft hyperrings.

Research in the area of complex fuzzy algebra is still in its infancy. The study of the complex fuzzy algebraic theory was initiated by Al-Husban et al. $[37,38]$ through the introduction of the algebraic structures of complex fuzzy subrings and complex fuzzy rings in [37,38], respectively. Al-Husban and Salleh [39], then, defined the notion of a complex fuzzy group, which is defined in a complex fuzzy space, instead of an ordinary universe of discourse. Alsarahead and Ahmad [40,41], then, proposed the structures of complex fuzzy subgroups and complex fuzzy soft groups in [40,41], respectively. To the best of our knowledge, these are the only published works in this area of research at present.

The aim of this section is to establish the novel concept of Complex Intuitionistic Fuzzy Soft groups (CIFS-groups) in the Rosenfelds sense (i.e., using the concept of a fuzzy subgroup of a group defined by Rosenfeld [42]). The properties and structural characteristics of the proposed algebraic structures are examined and, subsequently, verified.

Henceforth, symbol $G$ will be used to denote a group.

Definition 3.1 [19]. Let $(\mathcal{F}, E)$ be a non-null soft set on $G$. Then, $(\mathcal{F}, E)$ is said to be a soft group on $G$ if $\mathcal{F}(\varepsilon) \leqslant G$ for all $\varepsilon \in \mathscr{S}(\mathcal{F}, E)$.

Remark. As in classical group theory, a null set cannot be a group, and a null soft set on $G$ is not a soft group on $G$. 
Now, define the notion of a complex intuitionistic fuzzy subgroup of group $G$ and, then, use it to define the notion of a complex intuitionistic fuzzy soft group of a group $G$.

Definition 3.2. Let $M=\left(x, \mu_{M}(x), \nu_{M}(x)\right): x \in G$ be a complex intuitionistic fuzzy set on $G$. Then, $M$ is said to be a Complex Intuitionistic Fuzzy subgroup (CIF-subgroup) of $G$, if the following conditions hold for all $x, y \in G$ :

(i) $\mu_{M}(x y) \geqslant \min \left\{\mu_{M}(x), \mu_{M}(y)\right\}$;

(ii) $\nu_{M}(x y) \leqslant \max \left\{\nu_{M}(x), \nu_{M}(y)\right\}$;

(iii) $\mu_{M}\left(x^{-1}\right) \geqslant \mu_{M}(x)$;

(iv) $\nu_{M}\left(x^{-1}\right) \leqslant \nu_{M}(x)$.

Moreover, let $M$ and $N$ be two complex intuitionistic fuzzy subgroups of $G$ with $M \subseteq N$. In this case, $M$ is said to be a complex intuitionistic fuzzy subgroup of $N$.

Definition 3.3. Let $(\widetilde{\mathcal{F}}, E) \in \operatorname{CIFSS}(G)$. Then, $(\widetilde{\mathcal{F}}, E)$ is said to be a Complex Intuitionistic Fuzzy Soft group (CIFS-group) on $G$ if $\widetilde{\mathcal{F}}(\varepsilon)$ is a complex intuitionistic fuzzy subgroup of $G$ for all $\varepsilon \in \mathscr{S}(\widetilde{\mathcal{F}}, E)$.

In all that follows, $\operatorname{CIFSG}(G)$ denotes the collection of all complex intuitionistic fuzzy soft groups on a group $G$, and $(\widetilde{\mathcal{F}}, E) \in \operatorname{CIFSG}(G)$ denotes that $(\widetilde{\mathcal{F}}, E)$ is a complex intuitionistic fuzzy soft group on $G$.

Example 3.1. Consider the case where $G$ is the symmetric group of order 3 , that is, $G=S_{3}=$ $\{1,(12),(23),(13),(123),(132)\}$. Next, consider a set of parameters $E=\{a, b\}$. Herein, $\mu_{1}=0.4 \mathrm{e}^{\mathrm{i}}, \mu_{2}=0.4 \mathrm{e}^{2 \mathrm{i}}$, $\mu_{3}=0.7 \mathrm{e}^{2 \mathrm{i}} ;$ as well as $\nu_{1}=0.2 \mathrm{e}^{4 \mathrm{i}}, \nu_{2}=0.1 \mathrm{e}^{4 \mathrm{i}}$, $\nu_{3}=0.1 \mathrm{e}^{3 \mathrm{i}}$ are defined. Note that $\mu_{1} \leqslant \mu_{2} \leqslant \mu_{3}$ and $\nu_{1} \geqslant \nu_{2} \geqslant \nu_{3}$. Now, two CIFSSs of $G$ are considered, which are defined as follows:

(i) $(\widetilde{\mathcal{F}}, E)=\{\widetilde{\mathcal{F}}(a), \widetilde{\mathcal{F}}(b)\}$, where:

$$
\widetilde{\mathcal{F}}(a)=\left\{\begin{array}{c}
\left(1, \mu_{3}, \nu_{3}\right),\left((12), \mu_{1}, \nu_{1}\right), \\
\left((13), \mu_{1}, \nu_{1}\right),\left((23), \mu_{1}, \nu_{1}\right), \\
\left((123), \mu_{2}, \nu_{2}\right),\left((132), \mu_{2}, \nu_{2}\right)
\end{array}\right\}
$$

and:

$$
\widetilde{\mathcal{F}}(b)=\left\{\begin{array}{c}
\left(1, \mu_{3}, \nu_{3}\right),\left((12), \mu_{2}, \nu_{2}\right), \\
\left((13), \mu_{1}, \nu_{1}\right),\left((23), \mu_{1}, \nu_{1}\right), \\
\left((123), \mu_{1}, \nu_{1}\right),\left((132), \mu_{1}, \nu_{1}\right)
\end{array}\right\},
$$

(ii) $(\widetilde{\mathcal{G}}, E)=\{\widetilde{\mathcal{G}}(a), \widetilde{\mathcal{G}}(b)\}$, where:

$$
\widetilde{\mathcal{G}}(a)=\widetilde{\mathcal{F}}(a),
$$

and:

$$
\widetilde{\mathcal{G}}(b)=\left\{\begin{array}{c}
\left(1, \mu_{1}, \nu_{1}\right),\left((12), \mu_{2}, \nu_{2}\right), \\
\left((13), \mu_{2}, \nu_{2}\right),\left((23), \mu_{1}, \nu_{1}\right), \\
\left((123), \mu_{1}, \nu_{1}\right),\left((132), \mu_{1}, \nu_{1}\right)
\end{array}\right\} .
$$

Accordingly, it can be verified that $(\widetilde{\mathcal{F}}, E) \in$ $\operatorname{CIFSS}(G)$, whereas $(\widetilde{\mathcal{G}}, E) \notin \operatorname{CIFSS}(G)$.

Definition 3.4. Let $\left(\widetilde{\mathcal{F}}_{1}, E_{1}\right),\left(\tilde{\mathcal{F}}_{2}, E_{2}\right) \in \operatorname{CIFSS}(G)$. Then, $\left(\widetilde{\mathcal{F}}_{1}, E_{1}\right)$ is said to be a Complex Intuitionistic Fuzzy Soft subgroup (CIFS-subgroup) of $\left(\widetilde{\mathcal{F}}_{2}, E_{2}\right)$ if the following conditions are satisfied:

(i) $E_{1} \subseteq E_{2}$;

(ii) For all $\varepsilon \in E_{1}, \widetilde{\mathcal{F}}_{1}(\varepsilon)$ is a complex intuitionistic fuzzy subgroup of $\left(\widetilde{\mathcal{F}}_{2} \varepsilon\right)$.

Proposition 3.1. Let $(\widetilde{\mathcal{F}}, E) \in \operatorname{CIFSS}(G)$ and $1_{G}$ be the identity element of $G$. Then, the following results hold for all $\varepsilon \in E$ and for all $x \in G$ :

(i) $\mu_{\tilde{\mathcal{F}}(\varepsilon)}\left(x^{-1}\right)=\mu_{\widetilde{\mathcal{F}}(\varepsilon)}(x)$ and $\nu_{\tilde{\mathcal{F}}(\varepsilon)}\left(x^{-1}\right)=\nu_{\widetilde{\mathcal{F}}(\varepsilon)}(x)$,

(ii) $\mu_{\widetilde{\mathcal{F}}(\varepsilon)}\left(1_{G}\right) \geqslant \mu_{\widetilde{\mathcal{F}}(\varepsilon)}(x)$ and $\nu_{\widetilde{\mathcal{F}}(\varepsilon)}\left(1_{G}\right) \leqslant \nu_{\widetilde{\mathcal{F}}(\varepsilon)}(x)$.

Proof. Let $\varepsilon \in E$ and $x \in G$. By Definition 3.3, $\widetilde{\mathcal{F}}(\varepsilon)$ is a CIFS-subgroup of $G$, which enables us to utilize Definition 3.2 for proving both (i) and (ii):

(i) Both $\mu_{\widetilde{\mathcal{F}}(\varepsilon)}\left(x^{-1}\right) \geqslant \mu_{\widetilde{\mathcal{F}}(\varepsilon)}(x)$ and $\nu_{\widetilde{\mathcal{F}}(\varepsilon)}\left(x^{-1}\right) \leqslant$ $\nu_{\tilde{\mathcal{F}}(\varepsilon)}(x)$ directly follow from Definition 3.2. Since $x \in G$, we also have $x^{-1} \in G$. Thus, it follows that:

$$
\mu_{\widetilde{\mathcal{F}}(\varepsilon)}(x)=\mu_{\widetilde{\mathcal{F}}(\varepsilon)}\left(\left(x^{-1}\right)^{-1}\right) \geqslant \mu_{\widetilde{\mathcal{F}}(\varepsilon)}\left(x^{-1}\right),
$$

and:

$$
\nu_{\widetilde{\mathcal{F}}(\varepsilon)}(x)=\nu_{\widetilde{\mathcal{F}}(\varepsilon)}\left(\left(x^{-1}\right)^{-1}\right) \leqslant \nu_{\widetilde{\mathcal{F}}(\varepsilon)}\left(x^{-1}\right),
$$

due to Definition 3.2.

(ii) Note that $1_{G}=x x^{-1}$; thus, the conditions $\mu_{\tilde{\mathcal{F}}(\varepsilon)}\left(1_{G}\right) \geqslant \min \left\{\mu_{\widetilde{\mathcal{F}}(\varepsilon)}(x), \mu_{\tilde{\mathcal{F}}(\varepsilon)}\left(x^{-1}\right)\right\}$, and $\nu_{\tilde{\mathcal{F}}(\varepsilon)}$ $\left(1_{G}\right) \leqslant \max \left\{\nu_{\tilde{\mathcal{F}}(\varepsilon)}(x), \nu_{\tilde{\mathcal{F}}(\varepsilon)}\left(x^{-1}\right)\right\}$, follow from Definition 3.2. By (i), we have:

$$
\begin{aligned}
\min & \left\{\mu_{\tilde{\mathcal{F}}(\varepsilon)}(x), \mu_{\tilde{\mathcal{F}}(\varepsilon)}\left(x^{-1}\right)\right\} \\
& =\min \left\{\mu_{\tilde{\mathcal{F}}(\varepsilon) M}(x), \mu_{\tilde{\mathcal{F}}(\varepsilon)}(x)\right\}=\mu_{\tilde{\mathcal{F}}(\varepsilon)}(x),
\end{aligned}
$$


and:

$$
\begin{aligned}
\max & \left\{\nu_{\widetilde{\mathcal{F}}(\varepsilon)}(x), \nu_{\widetilde{\mathcal{F}}(\varepsilon)}\left(x^{-1}\right)\right\} \\
& =\max \left\{\nu_{\widetilde{\mathcal{F}}(\varepsilon)}(x), \nu_{\widetilde{\mathcal{F}}(\varepsilon)}(x)\right\}=\nu_{\widetilde{\mathcal{F}}(\varepsilon)}(x) .
\end{aligned}
$$

This completes the proof.

Proposition 3.2. Let $(\tilde{\mathcal{F}}, E) \in \operatorname{CIFSS}(G)$. Then, $(\tilde{\mathcal{F}}, E) \in \operatorname{CIFSG}(G)$ if and only if the following conditions are satisfied for all $\varepsilon \in E$ and for all $x, y \in G$ :

(i) $\mu_{\widetilde{\mathcal{F}}(\varepsilon)}\left(x y^{-1}\right) \geqslant \min \left\{\mu_{\widetilde{\mathcal{F}}(\varepsilon)}(x), \mu_{\widetilde{\mathcal{F}}(\varepsilon)}(y)\right\}$;

(ii) $\nu_{\widetilde{\mathcal{F}}(\varepsilon)}\left(x y^{-1}\right) \leqslant \max \left\{\nu_{\widetilde{\mathcal{F}}(\varepsilon)}(x), \nu_{\widetilde{\mathcal{F}}(\varepsilon)}(y)\right\}$.

Proof. $(\Rightarrow)$ Suppose that $(\widetilde{\mathcal{F}}, E) \in \operatorname{CIFSG}(G)$.

Let $\varepsilon \in E$ and $x, y \in G$. Then, $y^{-1} \in G$ too, and based on Definition 3.3, $\widetilde{\mathcal{F}}(\varepsilon)$ is a CIF-subgroup of $G$. The conditions:

$$
\mu_{\widetilde{\mathcal{F}}(\varepsilon)}\left(x y^{-1}\right) \geqslant \min \left\{\mu_{\widetilde{\mathcal{F}}(\varepsilon)}(x), \mu_{\widetilde{\mathcal{F}}(\varepsilon)}\left(y^{-1}\right)\right\},
$$

and:

$$
\nu_{\widetilde{\mathcal{F}}(\varepsilon)}\left(x y^{-1}\right) \leqslant \max \left\{\nu_{\widetilde{\mathcal{F}}(\varepsilon)}(x), \nu_{\widetilde{\mathcal{F}}(\varepsilon)}\left(y^{-1}\right)\right\},
$$

directly follow from Definition 3.2. Similarly, we have $\mu_{\widetilde{\mathcal{F}}(\varepsilon)}\left(y^{-1}\right) \geqslant \mu_{\widetilde{\mathcal{F}}(\varepsilon)}(y)$ and $\nu_{\widetilde{\mathcal{F}}(\varepsilon)}\left(y^{-1}\right) \leqslant \nu_{\widetilde{\mathcal{F}}(\varepsilon)}(y)$, which implies that:

$$
\begin{aligned}
\min & \left\{\mu_{\widetilde{\mathcal{F}}(\varepsilon)}(x), \mu_{\widetilde{\mathcal{F}}(\varepsilon)}\left(y^{-1}\right)\right\} \\
& \geqslant \min \left\{\mu_{\widetilde{\mathcal{F}}(\varepsilon)}(x), \mu_{\widetilde{\mathcal{F}}(\varepsilon)}(y)\right\},
\end{aligned}
$$

and:

$$
\begin{aligned}
\max & \left\{\nu_{\widetilde{\mathcal{F}}(\varepsilon)}(x), \nu_{\widetilde{\mathcal{F}}(\varepsilon)}\left(y^{-1}\right)\right\} \\
& \leqslant \max \left\{\nu_{\widetilde{\mathcal{F}}(\varepsilon)}(x), \nu_{\widetilde{\mathcal{F}}(\varepsilon)}(y)\right\} .
\end{aligned}
$$

Thus, conditions (i) and (ii) now hold.

$(\Leftarrow)$ Suppose that conditions (i) and (ii) are satisfied for all $\varepsilon \in E$ and for all $x, y \in G$.

By considering the case $x=y$, we have:

$$
\begin{aligned}
& \mu_{\tilde{\mathcal{F}}(\varepsilon)}\left(1_{G}\right)=\mu_{\tilde{\mathcal{F}}(\varepsilon)}\left(y y^{-1}\right) \\
& \quad \geqslant \min \left\{\mu_{\widetilde{\mathcal{F}}(\varepsilon)}(y), \mu_{\widetilde{\mathcal{F}}(\varepsilon)}(y)\right\}=\mu_{\widetilde{\mathcal{F}}(\varepsilon)}(y),
\end{aligned}
$$

and:

$$
\begin{aligned}
& \nu_{\widetilde{\mathcal{F}}(\varepsilon)}\left(1_{G}\right)=\nu_{\widetilde{\mathcal{F}}(\varepsilon)}\left(x x^{-1}\right) \\
& \quad \leqslant \max \left\{\nu_{\widetilde{\mathcal{F}}(\varepsilon)}(x), \nu_{\widetilde{\mathcal{F}}(\varepsilon)}(x)\right\}=\nu_{\widetilde{\mathcal{F}}(\varepsilon)}(x) .
\end{aligned}
$$

These imply that:

$$
\begin{aligned}
\mu_{\widetilde{\mathcal{F}}(\varepsilon)}\left(y^{-1}\right)=\mu_{\widetilde{\mathcal{F}}(\varepsilon)}\left(1_{G} y^{-1}\right) \\
\quad \geqslant \min \left\{\mu_{\widetilde{\mathcal{F}}(\varepsilon)}\left(1_{G}\right), \mu_{\widetilde{\mathcal{F}}(\varepsilon)}(y)\right\}=\mu_{\widetilde{\mathcal{F}}(\varepsilon)}(y),
\end{aligned}
$$

and:

$$
\begin{aligned}
\nu_{\widetilde{\mathcal{F}}(\varepsilon)} & \left(y^{-1}\right)=\nu_{\widetilde{\mathcal{F}}(\varepsilon)}\left(1_{G} y^{-1}\right) \\
& \leqslant \max \left\{\nu_{\widetilde{\mathcal{F}}(\varepsilon)}\left(1_{G}\right), \nu_{\widetilde{\mathcal{F}}(\varepsilon)}(y)\right\}=\nu_{\widetilde{\mathcal{F}}(\varepsilon)}(y) .
\end{aligned}
$$

By considering $y^{-1} \in G$, it follows that:

$$
\begin{aligned}
\mu_{\widetilde{\mathcal{F}}(\varepsilon)}(x y) & \geqslant \min \left\{\mu_{\widetilde{\mathcal{F}}(\varepsilon)}(x), \mu_{\widetilde{\mathcal{F}}(\varepsilon)}\left(y^{-1}\right)\right\} \\
& \geqslant \min \left\{\mu_{\widetilde{\mathcal{F}}(\varepsilon)}(x), \mu_{\widetilde{\mathcal{F}}(\varepsilon)}(y)\right\}, \\
\nu_{\widetilde{\mathcal{F}}(\varepsilon)}(x y) & \leqslant \max \left\{\nu_{\widetilde{\mathcal{F}}(\varepsilon)}(x), \nu_{\widetilde{\mathcal{F}}(\varepsilon)}\left(y^{-1}\right)\right\} \\
& \leqslant \max \left\{\nu_{\widetilde{\mathcal{F}}(\varepsilon)}(x), \nu_{\widetilde{\mathcal{F}}(\varepsilon)}(y)\right\} .
\end{aligned}
$$

Thus, $\widetilde{\mathcal{F}}(\varepsilon)$ is proved to be a CIF-subgroup of $G$ for all $\varepsilon \in E$; hence, it follows that $(\widetilde{\mathcal{F}}, E) \in \operatorname{CIFSG}(G)$.

Proposition 3.3. Let $(\tilde{\mathcal{F}}, E) \in \operatorname{CIFSG}(G)$, and $\alpha, \beta \in O_{1}$. If $(\widetilde{\mathcal{F}}, E)_{(\alpha, \beta)}$ is non-null, then it is a soft group of $G$.

Proof. The proof is straightforward.

Proposition 3.4. Let $S$ be a non-null subset of $G$ and $(\widetilde{\mathcal{F}}, E) \in \operatorname{CIFSS}(G)$, where $(\widetilde{\mathcal{F}}, E)$ is characteristic over $S$. IF $(\widetilde{\mathcal{F}}, E) \in \operatorname{CIFSG}(G)$, then $S$ is a classical subgroup of $G$.

Proof. Let $x, y \in S$. Then, by Definition 2.21, $\left\{\mu_{\widetilde{\mathcal{F}}(\varepsilon)}: \varepsilon \in E\right\}=\mu_{0}$ and $\left\{\nu_{\widetilde{\mathcal{F}}(\varepsilon)}: \varepsilon \in E\right\}=\nu_{0}$, in which there exist $\alpha, \beta \in \mathrm{O}_{1}$, with $\alpha \geqslant \beta$, such that $\mu_{0}(x)=\mu_{0}(y)=\alpha$ and $\nu_{0}(x)=\nu_{0}(y)=\beta$. Thus, it follows that $x, y \in \widetilde{\mathcal{F}}_{(\alpha, \beta)}(\varepsilon)$ for all $\varepsilon \in E$, which further implies that $\widetilde{\mathcal{F}}_{(\alpha, \beta)}(\varepsilon)$ is not empty for all $\varepsilon \in E$. Therefore, we have $\mathscr{S}(\widetilde{\mathcal{F}}, E)_{(\alpha, \beta)}=E$. Since $\mathscr{S}(\widetilde{\mathcal{F}}, E)_{(\alpha, \beta)}$ is not empty, $(\widetilde{\mathcal{F}}, E)_{(\alpha, \beta)}$ is non-null, and $(\widetilde{\mathcal{F}}, E)_{(\alpha, \beta)}$ is, therefore, a soft group of $G$.

Take $\varepsilon_{0} \in E$. Based on Definition 3.1, it follows that $\widetilde{\mathcal{F}}_{(\alpha, \beta)}\left(\varepsilon_{0}\right)$ is a subgroup of $G$; therefore, we have $x y^{-1} \in \widetilde{\mathcal{F}}_{(\alpha, \beta)}\left(\varepsilon_{0}\right)$. Then, by Definition 2.20, $\mu_{\widetilde{\mathcal{F}}\left(\varepsilon_{0}\right)}\left(x y^{-1}\right) \geqslant \alpha$ and $\nu_{\widetilde{\mathcal{F}}\left(\varepsilon_{0}\right)}\left(x y^{-1}\right) \leqslant \beta$.

Recall that $\left\{\mu_{\widetilde{\mathcal{F}}(\varepsilon)}: \varepsilon \in E\right\}=\mu_{0}$ and $\left\{\nu_{\widetilde{\mathcal{F}}(\varepsilon)}: \varepsilon \in\right.$ $E\}=\nu_{0}$. As a result, $\mu_{0}\left(x y^{-1}\right) \geqslant \alpha$ and $\nu_{0}\left(x y^{-1}\right) \leqslant \beta$. 
We now show that both $\mu_{0}\left(x y^{-1}\right)=\alpha$ and $\nu_{0}\left(x y^{-1}\right)=\beta$, which in turn implies that $x y^{-1} \in S$. We write $\alpha=r \mathrm{e}^{\mathrm{i} \omega}$ and $\beta=\tau \mathrm{e}^{\mathrm{i} \psi}$, for some $r, \tau \in$ $[0,1]$ and $\omega, \psi \in(0,2 \pi]$. Then, $\omega+\psi \in\{2 \pi, 4 \pi\}$ follows because of Definition 2.21. Furthermore, recall that $r+\tau=1$ (and, thus, $|\alpha|+|\beta|=1$ ) due to Proposition 2.1.

(a) By Definition 2.21, it is either $\mu_{0}\left(x y^{-1}\right)=\alpha$ or $\mu_{0}\left(x y^{-1}\right)=1 \sim \alpha$. Suppose that $\mu_{0}\left(x y^{-1}\right)=1 \sim$ $\alpha$; then, $1 \sim \alpha \geqslant \alpha$. Since $1 \sim \alpha=(1-r) \mathrm{e}^{\mathrm{i} \omega^{\prime}} \geqslant$ $r \mathrm{e}^{\mathrm{i} \omega}=\alpha$, it follows that $|\beta|=1-|\alpha|=|1 \sim \alpha| \geqslant$ $|\alpha| \geqslant|\beta|$. This further implies that $|1 \sim \alpha|=|\alpha|$ and, therefore, $1-r=r$. Therefore, we now have $1 \sim \alpha=r \mathrm{e}^{\mathrm{i} \omega^{\prime}}$, and there are two cases to consider:

(i) If $\omega=2 \pi$, then $\omega^{\prime}=\omega$ and, therefore, $1 \sim$ $\alpha=r \mathrm{e}^{\mathrm{i} \omega^{\prime}}=r \mathrm{e}^{\mathrm{i} \omega}=\alpha$. Hence, $\mu_{0}\left(x y^{-1}\right)=\alpha$ follows;

(ii) If $\omega<2 \pi$, we have both $\omega^{\prime}=(2 \pi-\omega)$ and $\omega+\psi=2 \pi$. Note that $\omega \geqslant \psi$ because of $\alpha \geqslant \beta$. As a result, $\omega \geqslant \pi$ follows. On the other hand, since $1 \sim \alpha=r \mathrm{e}^{\mathrm{i}(2 \pi-\omega)} \geqslant r \mathrm{e}^{\mathrm{i} \omega}=$ $\alpha$, we have $(2 \pi-\omega) \geqslant \omega$, which implies that $\pi \geqslant \omega$. Thus, we now have $\omega=\pi$, resulting in $\omega^{\prime}=\pi=\omega$ and, therefore, $1 \sim \alpha=r \mathrm{e}^{\mathrm{i} \omega^{\prime}}=$ $r \mathrm{e}^{\mathrm{i} \omega}=\alpha$. Hence, $\mu_{0}\left(x y^{-1}\right)=\alpha$ again follows.

(b) Based on Definition 2.21, it is either $\nu_{0}\left(x y^{-1}\right)=\beta$ or $\nu_{0}\left(x y^{-1}\right)=1 \sim \beta$. Suppose that $\nu_{0}\left(x y^{-1}\right)=$ $1 \sim \beta$; then, $1 \sim \beta \leqslant \beta$. Since $1 \sim \beta=(1-$ $\tau) \mathrm{e}^{\mathrm{i} \psi^{\prime}} \leqslant \tau \mathrm{e}^{\mathrm{i} \psi}=\beta$, it follows that $|\alpha|=1-|\beta|=$ $|1 \sim \beta| \leqslant|\beta| \leqslant|\alpha|$. This further implies that $|1 \sim \beta|=|\beta|$ and, therefore, $1-\tau=\tau$. Thus, we now have $1 \sim \beta=\tau \mathrm{e}^{\mathrm{i} \psi^{\prime}}$; there are two cases to consider:

(i) If $\psi=2 \pi$, then $\psi^{\prime}=\psi$ and, therefore, $1 \sim$ $\beta=\tau \mathrm{e}^{\mathrm{i} \psi^{\prime}}=\tau \mathrm{e}^{\mathrm{i} \psi}=\beta$. Hence, $\nu_{0}\left(x y^{-1}\right)=\beta$ follows;

(ii) If $\psi<2 \pi$, we have both $\psi^{\prime}=(2 \pi-\psi)$ and $\omega+\psi=2 \pi$. Note that $\omega \leqslant \psi$ because of $\beta \leqslant$ $\alpha$. As a result, $\psi \leqslant \pi$ follows. On the other hand, since $1 \sim \beta=\tau \mathrm{e}^{\mathrm{i}(2 \pi-\psi)} \geqslant \tau \mathrm{e}^{\mathrm{i} \psi}=\beta$, $(2 \pi-\psi) \leqslant \psi$ which implies that $\pi \leqslant \psi$. Thus, we now have $\psi=\pi$, resulting in $\psi^{\prime}=\pi=\psi$ and, therefore, $1 \sim \beta=\tau \mathrm{e}^{\mathrm{i} \psi^{\prime}}=\tau \mathrm{e}^{\mathrm{i} \psi}=\beta$. Hence, $\nu_{0}\left(x y^{-1}\right)=\beta$ again follows.

Therefore, we obtain $x y^{-1} \in S$ whenever $x, y \in S$. As such, it can be concluded that $S$ is a classical subgroup of $G$.

Theorem 3.1. Let $S$ be a non-null subset of $G$ and $(\widetilde{\mathcal{F}}, E) \in \operatorname{CIFSS}(G)$, where $(\widetilde{\mathcal{F}}, E)$ is characteristic over S. Then, $(\widetilde{\mathcal{F}}, E) \in \operatorname{CIFSG}(G)$ if and only if $S$ is a classical subgroup of $G$.

Proof. In Proposition 3.4, it has already been proved that $S$ is a classical subgroup of $G$ whenever $(\widetilde{\mathcal{F}}, E) \in$ $\operatorname{CIFSG}(G)$. Therefore, it suffices to prove that $(\widetilde{\mathcal{F}}, E)$ $\in \operatorname{CIFSG}(G)$ whenever $S$ is a classical subgroup of $G$.

Since $(\widetilde{\mathcal{F}}, E) \in \operatorname{CIFSS}(G)$ and $(\widetilde{\mathcal{F}}, E)$ is characteristic over $S$, by Definition 2.21, we have $\left\{\mu_{\tilde{\mathcal{F}}(\varepsilon)}: \varepsilon \in\right.$ $E\}=\mu_{0}$ and $\left\{\nu_{\tilde{\mathcal{F}}(\varepsilon)}: \varepsilon \in E\right\}=\nu_{0}$, in which:

$$
\mu_{0}(x)= \begin{cases}r \mathrm{e}^{\mathrm{i} \omega}, & x \in S \\ 1 \sim r \mathrm{e}^{\mathrm{i} \omega}, & x \in U-S\end{cases}
$$

and:

$$
\nu_{0}(x)= \begin{cases}\tau \mathrm{e}^{\mathrm{i} \psi}, & x \in S \\ 1 \sim \tau \mathrm{e}^{\mathrm{i} \psi}, & x \in U-S\end{cases}
$$

with $\omega+\psi \in\{2 \pi, 4 \pi\}$ and $r \mathrm{e}^{\mathrm{i} \omega} \geqslant \tau \mathrm{e}^{\mathrm{i} \psi}$.

Now, let $\varepsilon \in E$ and $x, y \in G$. Then both: $\left\{\mu_{\tilde{\mathcal{F}}(\varepsilon)}(x), \mu_{\widetilde{\mathcal{F}}(\varepsilon)}(y), \mu_{\tilde{\mathcal{F}}(\varepsilon)}\left(x y^{-1}\right)\right\} \subseteq\left\{r \mathrm{e}^{\mathrm{i} \omega}, 1 \sim r \mathrm{e}^{\mathrm{i} \omega}\right\}$ and $\left\{\nu_{\widetilde{\mathcal{F}}(\varepsilon)}(x), \nu_{\widetilde{\mathcal{F}}(\varepsilon)}(y), \nu_{\tilde{\mathcal{F}}(\varepsilon)}\left(x y^{-1}\right)\right\} \subseteq\left\{\tau \mathrm{e}^{\mathrm{i} \psi}, 1 \sim\right.$ $\left.\tau \mathrm{e}^{\mathrm{i} \psi}\right\}$. Furthermore, by Proposition 2.1, $r \mathrm{e}^{\mathrm{i} \omega} \geqslant$ $1 \sim r \mathrm{e}^{\mathrm{i} \omega}$ and $\tau \mathrm{e}^{\mathrm{i} \psi} \leqslant 1 \sim \tau \mathrm{e}^{\mathrm{i} \psi}$, which imply that $\min \left\{r \mathrm{e}^{\mathrm{i} \omega}, 1 \sim r \mathrm{e}^{\mathrm{i} \omega}\right\}=1 \sim r \mathrm{e}^{\mathrm{i} \omega}$ and $\max \left\{\tau \mathrm{e}^{\mathrm{i} \psi}, 1 \sim\right.$ $\left.\tau \mathrm{e}^{\mathrm{i} \psi}\right\}=1 \sim \tau \mathrm{e}^{\mathrm{i} \psi}$, respectively.

Without loss of generality, suppose that $x \in$ $G-S$. Then, $\mu_{\tilde{\mathcal{F}}(\varepsilon)}(x)=1 \sim r \mathrm{e}^{\mathrm{i} \omega}$ and $\nu_{\tilde{\mathcal{F}}(\varepsilon)}(x)=$ $1 \sim \tau \mathrm{e}^{\mathrm{i} \psi}$ which causes $\min \left\{\mu_{\tilde{\mathcal{F}}(\varepsilon)}(x), \mu_{\tilde{\mathcal{F}}(\varepsilon)}(y)\right\}=1$ $\sim r \mathrm{e}^{\mathrm{i} \omega}$ and $\max \left\{\nu_{\widetilde{\mathcal{F}}(\varepsilon)}(x), \nu_{\tilde{\mathcal{F}}(\varepsilon)}(y)\right\}=1 \sim \tau \mathrm{e}^{\mathrm{i} \psi}$, respectively. Since $\mu_{\widetilde{\mathcal{F}}(\varepsilon)}\left(x y^{-1}\right) \in\left\{r \mathrm{e}^{\mathrm{i} \omega}, 1 \sim r \mathrm{e}^{\mathrm{i} \omega}\right\}$ and $\nu_{\tilde{\mathcal{F}}(\varepsilon)}\left(x y^{-1}\right) \in\left\{\tau \mathrm{e}^{\mathrm{i} \psi}, 1 \sim \tau \mathrm{e}^{\mathrm{i} \psi}\right\}$, we conclude that:

$$
\begin{aligned}
\mu_{\widetilde{\mathcal{F}}(\varepsilon)}\left(x y^{-1}\right) & \geqslant \min \left\{r \mathrm{e}^{\mathrm{i} \omega}, 1 \sim r \mathrm{e}^{\mathrm{i} \omega}\right\}=1 \sim r \mathrm{e}^{\mathrm{i} \omega} \\
& =\min \left\{\mu_{\widetilde{\mathcal{F}}(\varepsilon)}(x), \mu_{\widetilde{\mathcal{F}}(\varepsilon)}(y)\right\}
\end{aligned}
$$

and:

$$
\begin{aligned}
\nu_{\widetilde{\mathcal{F}}(\varepsilon)}\left(x y^{-1}\right) & \leqslant \max \left\{\tau \mathrm{e}^{\mathrm{i} \psi}, 1 \sim \tau \mathrm{e}^{\mathrm{i} \psi}\right\}=1 \sim \tau \mathrm{e}^{\mathrm{i} \psi} \\
& =\max \left\{\nu_{\widetilde{\mathcal{F}}(\varepsilon)}(x), \nu_{\widetilde{\mathcal{F}}(\varepsilon)}(y)\right\} .
\end{aligned}
$$

Now, let $x, y \in S$. Since $S$ is a classical subgroup of $G$, $x y^{-1} \in S$; therefore, it follows that:

$$
\left\{\mu_{\widetilde{\mathcal{F}}(\varepsilon)}(x), \mu_{\widetilde{\mathcal{F}}(\varepsilon)}(y), \mu_{\widetilde{\mathcal{F}}(\varepsilon)}\left(x y^{-1}\right)\right\} \subseteq\left\{r \mathrm{e}^{\mathrm{i} \omega}\right\},
$$

and:

$$
\left\{\nu_{\widetilde{\mathcal{F}}(\varepsilon)}(x), \nu_{\widetilde{\mathcal{F}}(\varepsilon)}(y), \nu_{\widetilde{\mathcal{F}}(\varepsilon)}\left(x y^{-1}\right)\right\} \subseteq\left\{\tau \mathrm{e}^{\mathrm{i} \psi}\right\} .
$$

As a result, we have:

$$
\mu_{\widetilde{\mathcal{F}}(\varepsilon)}\left(x y^{-1}\right)=r \mathrm{e}^{\mathrm{i} \omega}=\min \left\{\mu_{\widetilde{\mathcal{F}}(\varepsilon)}(x), \mu_{\widetilde{\mathcal{F}}(\varepsilon)}(y)\right\},
$$

and: 


$$
\nu_{\widetilde{\mathcal{F}}(\varepsilon)}\left(x y^{-1}\right)=\tau \mathrm{e}^{\mathrm{i} \psi}=\max \left\{\nu_{\widetilde{\mathcal{F}}(\varepsilon)}(x), \nu_{\widetilde{\mathcal{F}}(\varepsilon)}(y)\right\}
$$

Hence, the conditions:

$$
\mu_{\tilde{\mathcal{F}}(\varepsilon)}\left(x y^{-1}\right) \geqslant \min \left\{\mu_{\tilde{\mathcal{F}}(\varepsilon)}(x), \mu_{\tilde{\mathcal{F}}(\varepsilon)}(y)\right\}
$$

and:

$$
\nu_{\widetilde{\mathcal{F}}(\varepsilon)}\left(x y^{-1}\right) \leqslant \max \left\{\nu_{\widetilde{\mathcal{F}}(\varepsilon)}(x), \nu_{\widetilde{\mathcal{F}}(\varepsilon)}(y)\right\}
$$

are shown to be satisfied for all $\varepsilon \in E$ and $x, y \in G$. This proves that $(\widetilde{\mathcal{F}}, E) \in \operatorname{CIFSG}(G)$.

Theorem 3.2. Let $(\tilde{\mathcal{F}}, E) \in \operatorname{CIFSS}(G)$, where $(\widetilde{\mathcal{F}}, E)$ is non-null. Then, the following statements are equivalent:

(i) $(\widetilde{\mathcal{F}}, E) \in \operatorname{CIFSG}(G)$;

(ii) For all $\alpha, \beta \in O_{1}$, either $(\widetilde{\mathcal{F}}, E)_{(\alpha, \beta)}$ is null, or $(\widetilde{\mathcal{F}}, E)_{(\alpha, \beta)}$ is a soft group of $G$.

\section{Proof.}

(i) $\Rightarrow$ (ii) Take any arbitrary $\alpha, \beta \in \mathrm{O}_{1}$. By Proposition 3.3 , if $(\widetilde{\mathcal{F}}, E)_{(\alpha, \beta)}$ is non-null, then it is a soft group of $G$. Thus, statement (ii) is proved true;

(ii) $\Rightarrow$ (i) Let $\varepsilon \in E$ and $x, y \in G$, and note that $\mu_{\widetilde{\mathcal{F}}(\varepsilon)}(x), \mu_{\widetilde{\mathcal{F}}(\varepsilon)}(y), \nu_{\widetilde{\mathcal{F}}(\varepsilon)}(x), \nu_{\widetilde{\mathcal{F}}(\varepsilon)}(y) \in \mathrm{O}_{1}$.

Take $\alpha=\min \left\{\mu_{\widetilde{\mathcal{F}}(\varepsilon)}(x), \mu_{\widetilde{\mathcal{F}}(\varepsilon)}(y)\right\}$ and $\beta=\max \{$ $\left.\nu_{\widetilde{\mathcal{F}}(\varepsilon)}(x), \nu_{\widetilde{\mathcal{F}}(\varepsilon)}(y)\right\}$. Then, we have $\mu_{\widetilde{\mathcal{F}}(\varepsilon)}(x), \mu_{\widetilde{\mathcal{F}}(\varepsilon)}(y)$ $\geqslant \alpha$ and $\nu_{\widetilde{\mathcal{F}}(\varepsilon)}(x), \nu_{\widetilde{\mathcal{F}}(\varepsilon)}(y) \leqslant \beta$, which means that $x, y \in \widetilde{\mathcal{F}}_{(\alpha, \beta)}(\varepsilon)$. This implies that $(\widetilde{\mathcal{F}}, E)_{(\alpha, \beta)}$ is not null; therefore, it is a soft group of $G$. Thus, we now have $\widetilde{\mathcal{F}}_{(\alpha, \beta)}(\varepsilon) \leqslant G$ and, therefore, $x y^{-1} \in \widetilde{\mathcal{F}}_{(\alpha, \beta)}(\varepsilon)$, which in turn implies that:

$$
\mu_{\widetilde{\mathcal{F}}(\varepsilon)}\left(x y^{-1}\right) \geqslant \alpha=\min \left\{\mu_{\widetilde{\mathcal{F}}(\varepsilon)}(x), \mu_{\widetilde{\mathcal{F}}(\varepsilon)}(y)\right\}
$$

and:

$$
\nu_{\widetilde{\mathcal{F}}(\varepsilon)}\left(x y^{-1}\right) \leqslant \beta=\max \left\{\nu_{\widetilde{\mathcal{F}}(\varepsilon)}(x), \nu_{\widetilde{\mathcal{F}}(\varepsilon)}(y)\right\} .
$$

Hence, by Proposition 3.2, statement (i) now follows.

Theorem 3.3. $\operatorname{Let}\left(\widetilde{\mathcal{F}}_{1}, E_{1}\right),\left(\widetilde{\mathcal{F}}_{2}, E_{2}\right) \in \operatorname{CIFSG}(G)$. Then, $\left(\widetilde{\mathcal{F}}_{1}, E_{1}\right) \widetilde{\cap}\left(\widetilde{\mathcal{F}}_{2}, E_{2}\right) \in \operatorname{CIFSG}(G)$ too.

Proof. The proof is straightforward by Definition 2.19 and is, therefore, omitted.

Remark. This property also holds for the restricted intersection operation between CIFSSs.

Definition 3.5. Let $U_{1}, U_{2}$ be two universal sets, $\varphi$ : $U_{1} \rightarrow U_{2}$ be a function, $E, B$ be two sets of parameters:
$(\widetilde{\mathcal{T}}, E) \in \operatorname{CIFSS}\left(U_{1}\right)$ and $(\widetilde{\mathcal{F}}, B) \in \operatorname{CIFSS}\left(U_{2}\right)$. Define $(\varphi(\tilde{\mathcal{T}}), E) \in \operatorname{CIFSS}\left(U_{2}\right)$ and $\left(\varphi^{-1}(\tilde{\mathcal{F}}), B\right) \in \operatorname{CIFSS}\left(U_{1}\right)$ as follows:

(i) $(\varphi(\widetilde{\mathcal{T}}), E)$ is such that for all $y \in U_{2}$ and $\varepsilon \in E$ :

$$
\begin{aligned}
& \mu_{\varphi(\widetilde{\mathcal{T}})(\varepsilon)}(y) \\
& =\max \left\{\left\{\mu_{\widetilde{\mathcal{T}}(\varepsilon)}(u): u \in U_{1}, \varphi(u)=y\right\} \cup\{0\}\right\},
\end{aligned}
$$

and:

$$
\begin{aligned}
& \nu_{\varphi(\tilde{\mathcal{T}})(\varepsilon)}(y) \\
& \quad=\min \left\{\left\{\nu_{\tilde{\mathcal{T}}_{(\varepsilon)}}(u): u \in U_{1}, \varphi(u)=y\right\} \cup\{1\}\right\} .
\end{aligned}
$$

(ii) $\left(\varphi^{-1}(\tilde{\mathcal{F}}), B\right)$ is such that, for all $x \in U_{1}$ and $s \in B$, $\mu_{\varphi^{-1}(\tilde{\mathcal{F}})(s)}(x)=\mu_{\tilde{\mathcal{F}}(s)}(\varphi(x))$ and $\nu_{\varphi^{-1}(\tilde{\mathcal{F}})(s)}(x)=$ $\nu_{\tilde{\mathcal{F}}(s)}(\varphi(x))$.

Theorem 3.4. Let $\varphi: G \rightarrow G^{\prime}$ be a surjective group homomorphism. Let $(\widetilde{\mathcal{T}}, E) \in \operatorname{CIFSG}(G)$ and $(\widetilde{\mathcal{F}}, B) \in$ $\operatorname{CIFSG}\left(G^{\prime}\right)$. Then:

(i) $(\varphi(\tilde{\mathcal{T}}), E) \in \operatorname{CIFSG}\left(G^{\prime}\right)$ provided that:

$$
\begin{aligned}
& \max \left\{\min \left\{\mu_{\widetilde{\mathcal{T}}(\varepsilon)}(p), \mu_{\widetilde{\mathcal{T}}(\varepsilon)}(q)\right\}: \begin{array}{l}
p, q \in G \\
\varphi(p)=x, \varphi(q)=y
\end{array}\right\} \\
& \geqslant \min \left\{\mu_{\widetilde{\mathcal{T}}(\varepsilon)}(x), \mu_{\widetilde{\mathcal{T}}(\varepsilon)}(y)\right\}
\end{aligned}
$$

and:

$$
\begin{aligned}
& \min \left\{\max \left\{\nu_{\widetilde{\mathcal{T}}(\varepsilon)}(p), \nu_{\widetilde{\mathcal{T}}(\varepsilon)}(q)\right\}: \begin{array}{l}
p, q \in G \\
\varphi(p)=x, \varphi(q)=y
\end{array}\right\} \\
& \leqslant \max \left\{\nu_{\widetilde{\mathcal{T}}(\varepsilon)}(x), \nu_{\widetilde{\mathcal{T}}(\varepsilon)}(y)\right\}
\end{aligned}
$$

for all $x, y \in G^{\prime}$;

(ii) $\left(\varphi^{-1}(\widetilde{\mathcal{F}}), B\right) \in \operatorname{CIFSG}(G)$.

\section{Proof.}

(i) Let $x, y \in G^{\prime}$ and $\varepsilon \in E$. Then, by Definition 3.5 , we have $(\varphi(\tilde{\mathcal{T}}), E) \in \operatorname{CIFSS}\left(G^{\prime}\right)$, where $\mu_{\varphi(\tilde{\mathcal{T}})(\varepsilon)}\left(x y^{-1}\right)=\max \left\{\left\{\mu_{\widetilde{\mathcal{T}}(\varepsilon)}(u): u \in G, \varphi(u)=\right.\right.$ $\left.\left.x y^{-1}\right\} \cup\{0\}\right\}$. Since $\varphi$ is surjective, we have:

$$
\begin{aligned}
\max & \left\{\left\{\mu_{\widetilde{\mathcal{T}}(\varepsilon)}(u): u \in G, \varphi(u)=x y^{-1}\right\} \cup\{0\}\right\} \\
& =\max \left\{\mu_{\widetilde{\mathcal{T}}(\varepsilon)}(u): u \in G, \varphi(u)=x y^{-1}\right\} .
\end{aligned}
$$

Moreover, since $\varphi$ is also a homomorphism, we have: 


$$
\begin{aligned}
& \max \left\{\mu_{\widetilde{\mathcal{T}}(\varepsilon)}(u): u \in G, \varphi(u)=x y^{-1}\right\} \\
& \geqslant \max \left\{\mu_{\widetilde{\mathcal{T}}(\varepsilon)}\left(p q^{-1}\right): p, q \in G, \varphi(p)\right. \\
& \quad=x, \varphi(q)=y\} .
\end{aligned}
$$

Since $(\widetilde{\mathcal{T}}, E) \in \operatorname{CIFSG}(G), \mu_{\widetilde{\mathcal{T}}(\varepsilon)}\left(p q^{-1}\right) \geqslant \min \{$ $\left.\mu_{\widetilde{\mathcal{T}}(\varepsilon)}(p), \mu_{\widetilde{\mathcal{T}}(\varepsilon)}(q)\right\}$ for all $p, q \in G$. This implies that:

$$
\begin{aligned}
& \max \left\{\mu_{\widetilde{\mathcal{T}}(\varepsilon)}\left(p q^{-1}\right): p, q \in G, \varphi(p)=x, \varphi(q)=y\right\} \\
& \geqslant \max \left\{\min \left\{\mu_{\widetilde{\mathcal{T}}(\varepsilon)}(p), \mu_{\widetilde{\mathcal{T}}(\varepsilon)}(q)\right\}: p, q\right. \\
& \quad \in G, \varphi(p)=x, \varphi(q)=y\} \\
& \quad \geqslant \min \left\{\mu_{\widetilde{\mathcal{T}}(\varepsilon)}(x), \mu_{\widetilde{\mathcal{T}}(\varepsilon)}(y)\right\} .
\end{aligned}
$$

Similarly, for the non-membership function, we have the following:

$$
\begin{aligned}
& \nu_{\varphi(\tilde{\mathcal{T}})(\varepsilon)}\left(x y^{-1}\right) \\
& =\min \left\{\left\{\nu_{\widetilde{\mathcal{T}}(\varepsilon)}(u): u \in G, \varphi(u)=x y^{-1}\right\} \cup\{0\}\right\} \\
& =\min \left\{\nu_{\widetilde{\mathcal{T}}(\varepsilon)}(u): u \in G, \varphi(u)=x y^{-1}\right\} \\
& \leqslant \min \left\{\nu_{\widetilde{\mathcal{T}}(\varepsilon)}\left(p q^{-1}\right): p, q \in G, \varphi(p)=x, \varphi(q)=y\right\} \\
& \leqslant \min \left\{\max \left\{\nu_{\widetilde{\mathcal{T}}(\varepsilon)}(p), \nu_{\widetilde{\mathcal{T}}(\varepsilon)}(q)\right\}: p, q\right. \\
& \quad \in G, \varphi(p)=x, \varphi(q)=y\} \\
& \leqslant \max \left\{\nu_{\widetilde{\mathcal{T}}(\varepsilon)}(x), \nu_{\widetilde{\mathcal{T}}(\varepsilon)}(y)\right\} .
\end{aligned}
$$

(ii) Let $x, y \in G$ and $s \in B$. Then, $\left(\varphi^{-1}(\widetilde{\mathcal{F}}), B\right) \in$ $\operatorname{CIFSS}(G)$ by Definition 3.5. As $(\widetilde{\mathcal{F}}, B) \in$ CIFSG $\left(G^{\prime}\right)$, by applying Proposition 3.2, alongside with Definition 3.5, we obtain the following:

$$
\begin{aligned}
& \mu_{\varphi^{-1}(\widetilde{\mathcal{F}})(s)}\left(x y^{-1}\right)=\mu_{\widetilde{\mathcal{F}}(s)}\left(\varphi\left(x y^{-1}\right)\right) \\
& =\mu_{\widetilde{\mathcal{F}}(s)}\left(\varphi(x)(\varphi(y))^{-1}\right) \\
& \geqslant \min \left\{\mu_{\widetilde{\mathcal{F}}(s)}(\varphi(x)), \mu_{\widetilde{\mathcal{F}}(s)}(\varphi(y))\right\} \\
& =\min \left\{\mu_{\varphi^{-1}(\widetilde{\mathcal{F}})(s)}(x), \mu_{\varphi^{-1}(\widetilde{\mathcal{F}})(s)}(y)\right\} \\
& \nu_{\varphi^{-1}(\widetilde{\mathcal{F}})(s)}\left(x y^{-1}\right)=\nu_{\widetilde{\mathcal{F}}(s)}\left(\varphi\left(x y^{-1}\right)\right)
\end{aligned}
$$

$$
\begin{aligned}
& =\nu_{\widetilde{\mathcal{F}}(s)}\left(\varphi(x)(\varphi(y))^{-1}\right) \\
& \leqslant \max \left\{\nu_{\widetilde{\mathcal{F}}(s)}(\varphi(x)), \nu_{\widetilde{\mathcal{F}}(s)}(\varphi(y))\right\} \\
& =\max \left\{\nu_{\varphi^{-1}(\tilde{\mathcal{F}})(s)}(x), \nu_{\varphi^{-1}(\tilde{\mathcal{F}})(s)}(y)\right\} .
\end{aligned}
$$

This completes the proof.

\section{Normal complex intuitionistic fuzzy soft groups}

In this section, the notion of CIFS-groups is extended by adding the normality condition to the existing conditions. Aygunoglu \& Aygun [20] introduced the conditions for normality in the context of fuzzy soft sets. Here, these conditions are generalized for normality to be compatible with the CIFSS model; subsequently, these conditions are used to define the notion of normal CIFS-groups.

The conditions for intuitionistic fuzzy soft normality are described in Lemma 4.1, whereas the notion of a normal CIFS-group is proposed in Definition 4.1.

Lemma 4.1. Let $(\widetilde{\mathcal{F}}, E) \in \operatorname{CIFSG}(G)$ and $\varepsilon \in E$. Then, the following statements are equivalent:

(i) $\mu_{\tilde{\mathcal{F}}(\varepsilon)}\left(x y x^{-1}\right) \geqslant \mu_{\tilde{\mathcal{F}}(\varepsilon)}(y)$ and $\nu_{\widetilde{\mathcal{F}}(\varepsilon)}\left(x y x^{-1}\right) \leqslant$ $\nu_{\widetilde{\mathcal{F}}(\varepsilon)}(y)$, for all $x, y \in G$;

(ii) $\mu_{\widetilde{\mathcal{F}}(\varepsilon)}\left(x y x^{-1}\right)=\mu_{\widetilde{\mathcal{F}}(\varepsilon)}(y)$ and $\nu_{\widetilde{\mathcal{F}}(\varepsilon)}\left(x y x^{-1}\right)=$ $\nu_{\widetilde{\mathcal{F}}(\varepsilon)}(y)$, for all $x, y \in G$;

(iii) $\mu_{\tilde{\mathcal{F}}(\varepsilon)}(x y)=\mu_{\widetilde{\mathcal{F}}(\varepsilon)}(y x)$ and $\nu_{\widetilde{\mathcal{F}}(\varepsilon)}(x y)=\nu_{\widetilde{\mathcal{F}}(\varepsilon)}(y x)$, for all $x, y \in G$.

\section{Proof.}

(i) $\Rightarrow$ (ii) Let $x, y \in G$. Then, $y=x^{-1}\left(x y x^{-1}\right)\left(x^{-1}\right)^{-1}$, and both $x^{-1}, x y x^{-1} \in G$. As a result, we have:

$$
\begin{aligned}
\mu_{\tilde{\mathcal{F}}(\varepsilon)}(y) & =\mu_{\tilde{\mathcal{F}}(\varepsilon)}\left(x^{-1}\left(x y x^{-1}\right)\left(x^{-1}\right)^{-1}\right) \\
& \geqslant \mu_{\tilde{\mathcal{F}}(\varepsilon)}\left(x y x^{-1}\right)
\end{aligned}
$$

and:

$$
\begin{aligned}
\nu_{\widetilde{\mathcal{F}}(\varepsilon)}(y) & =\nu_{\widetilde{\mathcal{F}}(\varepsilon)}\left(x^{-1}\left(x y x^{-1}\right)\left(x^{-1}\right)^{-1}\right) \\
& \leqslant \nu_{\widetilde{\mathcal{F}}(\varepsilon)}\left(x y x^{-1}\right) .
\end{aligned}
$$

Hence, statement (ii) now follows;

(ii) $\Rightarrow$ (iii) Let $x, y \in G$. Then, $x y=x(y x) x^{-1}$ and $y x \in$ $G$. As a result, we now have:

$$
\mu_{\widetilde{\mathcal{F}}(\varepsilon)}(x y)=\mu_{\widetilde{\mathcal{F}}(\varepsilon)}\left(x(y x) x^{-1}\right)=\mu_{\widetilde{\mathcal{F}}(\varepsilon)}(y x),
$$

and: 


$$
\nu_{\widetilde{\mathcal{F}}(\varepsilon)}(x y)=\nu_{\widetilde{\mathcal{F}}(\varepsilon)}\left(x(y x) x^{-1}\right)=\nu_{\widetilde{\mathcal{F}}(\varepsilon)}(y x) .
$$

Hence, statement (iii) now follows;

(iii) $\Rightarrow$ (i) Let $x, y \in G$. Then $\left(x^{-1}\right)(x y)=y$, where $x y, x^{-1} \in G$. Thus, we obtain:

$$
\mu_{\widetilde{\mathcal{F}}(\varepsilon)}\left((x y) x^{-1}\right)=\mu_{\widetilde{\mathcal{F}}(\varepsilon)}\left(x^{-1}(x y)\right)=\mu_{\widetilde{\mathcal{F}}(\varepsilon)}(y),
$$

and:

$$
\nu_{\widetilde{\mathcal{F}}(\varepsilon)}\left((x y) x^{-1}\right)=\nu_{\widetilde{\mathcal{F}}(\varepsilon)}\left(x^{-1}(x y)\right)=\nu_{\widetilde{\mathcal{F}}(\varepsilon)}(y) .
$$

Hence, statement (i) now follows.

Definition 4.1. Let $(\widetilde{\mathcal{F}}, E) \in \operatorname{CIFSG}(G)$. Then $(\tilde{\mathcal{F}}, E)$ is said to be a Normal Complex Intuitionistic Fuzzy Soft Group on $G((\widetilde{\mathcal{F}}, E) \in \operatorname{NCIFSG}(G))$, if the conditions:

$$
\mu_{\widetilde{\mathcal{F}}(\varepsilon)}\left(x y x^{-1}\right) \geqslant \mu_{\widetilde{\mathcal{F}}(\varepsilon)}(y),
$$

and:

$$
\nu_{\widetilde{\mathcal{F}}(\varepsilon)}\left(x y x^{-1}\right) \leqslant \nu_{\widetilde{\mathcal{F}}(\varepsilon)}(y),
$$

are satisfied for all $\varepsilon \in E$ and $x, y \in G$.

Example 4.1. Consider the example described in Example 3.1. We define an $(\widetilde{\mathcal{H}}, E) \in \operatorname{CIFSS}(G)$ as $(\widetilde{\mathcal{H}}, E)=\{\widetilde{\mathcal{H}}(a), \widetilde{\mathcal{H}}(b)\}$, where $\widetilde{\mathcal{H}}(a)=\widetilde{\mathcal{F}}(a)$, and $\widetilde{\mathcal{F}}(a)$ is as defined in Example 3.1. and:

$$
\widetilde{\mathcal{H}}(b)=\left\{\begin{array}{c}
\left(1, \mu_{3}, \nu_{3}\right),\left((12), \mu_{1}, \nu_{1}\right),\left((13), \mu_{1}, \nu_{1}\right), \\
\left((23), \mu_{1}, \nu_{1}\right),\left((123), \mu_{1}, \nu_{1}\right), \\
\left((132), \mu_{1}, \nu_{1}\right)
\end{array}\right\} .
$$

Then, $(\tilde{\mathcal{H}}, E) \in \operatorname{CIFSG}(G)$ and it also satisfies the conditions for normality described in Definition 4.1 . Hence, $(\widetilde{\mathcal{H}}, E) \in \operatorname{NCIFSG}(G)$.

Theorem 4.1. Let $(\widetilde{\mathcal{F}}, E) \in \operatorname{CIFSG}(G)$. Then, the following statements are equivalent:

(i) $(\tilde{\mathcal{F}}, E) \in \operatorname{NCIFSG}(G)$;

(ii) $\mu_{\widetilde{\mathcal{F}}(\varepsilon)}\left(x y x^{-1}\right) \geqslant \mu_{\widetilde{\mathcal{F}}(\varepsilon)}(y)$ and $\nu_{\widetilde{\mathcal{F}}(\varepsilon)}\left(x y x^{-1}\right) \leqslant$ $\nu_{\widetilde{\mathcal{F}}(\varepsilon)}(y)$, for all $\varepsilon \in E$ and $x, y \in G$;

(iii) $\mu_{\widetilde{\mathcal{F}}(\varepsilon)}\left(x y x^{-1}\right)=\mu_{\widetilde{\mathcal{F}}(\varepsilon)}(y)$ and $\nu_{\widetilde{\mathcal{F}}(\varepsilon)}\left(x y x^{-1}\right)=$ $\nu_{\widetilde{\mathcal{F}}(\varepsilon)}(y)$, for all $\varepsilon \in E$ and $x, y \in G$;

(iv) $\mu_{\tilde{\mathcal{F}}(\varepsilon)}(x y)=\mu_{\tilde{\mathcal{F}}(\varepsilon)}(y x)$ and $\nu_{\tilde{\mathcal{F}}(\varepsilon)}(x y)=$ $\nu_{\widetilde{\mathcal{F}}(\varepsilon)}(y x)$, for all $\varepsilon \in E$ and $x, y \in G$.

\section{Proof.}

(i) $\Rightarrow$ (ii) This follows directly from Definition 4.1;

(ii) $\Rightarrow$ (iii) and (iii) $\Rightarrow$ (iv) These follow directly from Lemma 4.1;

(iv) $\Rightarrow$ (i) Statement (ii) follows directly from Definition 4.1 and, therefore, statement (i) follows by Lemma 4.1.

Proposition 4.1. Let $(\widetilde{\mathcal{F}}, E) \in \operatorname{NCIFSG}(G)$ and $\emptyset \subset D \subseteq E$. Then $(\widetilde{\mathcal{F}}, D) \in \operatorname{NCIFSG}(G)$ as well.

Proof. Let $x, y \in G$. By Theorem 4.1, it follows that $\mu_{\tilde{\mathcal{F}}(\varepsilon)}\left(x y x^{-1}\right) \geqslant \mu_{\widetilde{\mathcal{F}}(\varepsilon)}(y)$ and $\nu_{\widetilde{\mathcal{F}}(\varepsilon)}\left(x y x^{-1}\right) \leqslant \nu_{\tilde{\mathcal{F}}(\varepsilon)}(y)$, for all $\varepsilon \in E$. Since $\emptyset \subset D \subseteq E$, such statement holds for all $\varepsilon \in D$ too. This completes the proof.

Theorem 4.2. Let:

$$
\left(\widetilde{\mathcal{F}}_{1}, E_{1}\right),\left(\widetilde{\mathcal{F}}_{2}, E_{2}\right) \in \operatorname{NCIFSG}(G) .
$$

Then:

$$
\left(\widetilde{\mathcal{F}}_{1}, E_{1}\right) \widetilde{\cap}\left(\widetilde{\mathcal{F}}_{2}, E_{2}\right) \in \operatorname{NCIFSG}(G) .
$$

Proof. The proof is straightforward by Definitions 2.14 and 4.1 .

Remark. Similar to Theorem 3.3, this property also holds for the restricted intersection operation between CIFSSs.

Theorem 4.3. Let $\varphi: G \rightarrow G^{\prime}$ be a surjective group homomorphism. Let $(\widetilde{\mathcal{T}}, E) \in \operatorname{NCIFSG}(G)$ and $(\widetilde{\mathcal{F}}, B) \in \operatorname{NCIFSG}\left(G^{\prime}\right)$. Then:

(i) $(\varphi(\widetilde{\mathcal{T}}), E) \in \operatorname{NCIFSG}\left(G^{\prime}\right)$ provided that:

$$
\begin{gathered}
\max \left\{\min \left\{\mu_{\widetilde{\mathcal{T}}(\varepsilon)}(p), \mu_{\widetilde{\mathcal{T}}(\varepsilon)}(q)\right\}: \begin{array}{l}
p, q \in G \\
\varphi(p)=x, \varphi(q)=y
\end{array}\right\} \\
\geqslant \min \left\{\mu_{\widetilde{\mathcal{T}}(\varepsilon)}(x), \mu_{\widetilde{\mathcal{T}}(\varepsilon)}(y)\right\},
\end{gathered}
$$

and:

$$
\begin{aligned}
& \quad \min \left\{\max \left\{\nu_{\widetilde{\mathcal{T}}(\varepsilon)}(p), \nu_{\widetilde{\mathcal{T}}(\varepsilon)}(q)\right\}: \begin{array}{l}
p, q \in G \\
\varphi(p)=x, \varphi(q)=y
\end{array}\right\} \\
& \quad \leqslant \max \left\{\nu_{\widetilde{\mathcal{T}}(\varepsilon)}(x), \nu_{\widetilde{\mathcal{T}}(\varepsilon)}(y)\right\} \\
& \quad \text { for all } x, y \in G^{\prime}, \\
& \text { (ii) } \quad\left(\varphi^{-1}(\widetilde{\mathcal{F}}), B\right) \in \operatorname{NCIFSG}(G) .
\end{aligned}
$$

Proof. The proof can be derived from Theorem 3.4, Lemma 4.1, and Definition 4.1.

\section{Conclusion}

This paper presented the initial theory of complex 
fuzzy algebra. We defined and developed the algebraic structures pertaining to groups and subgroups for the Complex Intuitionistic Fuzzy Soft Set (CIFSS) model. The notions of CIF-subgroups, CIFS-groups, and normal CIFS-groups were introduced. The fundamental properties and structural characteristics of these algebraic structures were then examined and verified. All of these were accomplished by carefully defining some important concepts pertaining to the structure of the CIFSS model and also carefully generalizing some of the well-known operations and relations that exist between intuitionistic fuzzy soft sets to be made compatible with the structure of the CIFSS model, in which the membership and non-membership functions are defined in terms of complex numbers. Furthermore, in this paper, we contextualized the phase term by using it to represent the different cycles of alternating groups, thereby proposing a new way of interpreting the phase term.

\section{Further direction of this work}

Our research in this area is still ongoing. We are currently in the midst of extending the CIFSG structure introduced in this paper to introduce more advanced algebraic structures, such as CIFS cyclic groups, abelian groups, dihedral groups, symmetric groups, and alternating groups, using the concepts and theory developed in this paper. The work presented in this paper can also be used as a basis to develop other algebraic theories of complex fuzzy based models.

\section{Acknowledgments}

Authors Quek and Selvachandran would like to gratefully acknowledge the financial assistance received from the Ministry of Education, Malaysia under grant no. FRGS/1/2017/STG06/UCSI/03/1 and UCSI University, Kuala Lumpur, Malaysia under grant no. ProjIn-FOBIS-014.

\section{References}

1. Zadeh, L.A. "Fuzzy sets", Information and Control, 8, pp. 338-353 (1965).

2. Atanassov, K.T. "Intuitionistic fuzzy sets", Fuzzy Sets and Systems, 20(1), pp. 87-96 (1986).

3. Molodtsov, D. "Soft set theory - First results", Computers and Mathematics with Applications, 37(4-5), pp. 19-31 (1999).

4. Maji, P.K., Biswas, R., and Roy, A.R. "Fuzzy soft sets", Journal of Fuzzy Mathematics, 3(9), pp. 589-602 (2001).

5. Maji, P.K., Biswas, R., and Roy, A.R. "Intuitionistic fuzzy soft sets", Journal of Fuzzy Mathematics, 9(3), pp. 677-692 (2001).
6. Yang, X., Lin, T.Y., Yang, J., Li, Y., and Yu, D. "Combination of interval-valued fuzzy set and soft set", Computers and Mathematics with Applications, 58, pp. 521-527 (2009).

7. Jiang, Y., Tang, Y., Chen, Q., Liu, H., and Tang, J. "Interval-valued intuitionistic fuzzy soft sets and their properties", Computers and Mathematics with Applications, 60, pp. 906-918 (2010).

8. Xu, W., Ma, J., Wang, S., and Hao, G. "Vague soft sets and their properties", Computers and Mathematics with Applications, 59, pp. 787-794 (2010).

9. Ramot, D., Milo, R., Friedman, M., and Kandel, A. "Complex fuzzy sets", IEEE Transactions on Fuzzy Systems, 10(2), pp. 171-186 (2002).

10. Kumar, T. and Bajaj, R.K. "On complex intuitionistic fuzzy soft sets with distance measures and entropies", Journal of Mathematics, 2014, pp. 1-12 (2014).

11. Feng, F., Young, B.J., and Zhao, X. "Soft semirings", Computers and Mathematics with Applications, 56, pp. 2621-2628 (2008).

12. Greenfield, S., Chiclana, F., and Dick, S. "Intervalvalued complex fuzzy logic", IEEE International Conference on Fuzzy Systems (FUZZ-IEEE), Vancouver, Canada, pp. 2014-2019 (2016).

13. Alkouri, A.U.M. and Salleh, A.R. "Complex intuitionistic fuzzy sets", AIP Conference Proceedings, 1482, pp. 464-470 (2012).

14. Selvachandran, G., Maji, P.K., Abed, I.E., and Salleh, A.R. "Complex vague soft sets and its distance measures", Journal of Intelligent and Fuzzy Systems, 31, pp. 55-68 (2016).

15. Selvachandran, G., Maji, P.K., Abed, I.E., and Salleh, A.R. "Relations between complex vague soft sets", Applied Soft Computing, 47, pp. 438-448 (2016).

16. Ramot, D., Friedman, M., Langholz, G., and Kandel, A. "Complex fuzzy logic", IEEE Transactions on Fuzzy Systems, 11(4), pp. 450-461 (2003).

17. Dick, S. "Toward complex fuzzy logic", IEEE Transactions on Fuzzy Systems, 13(3), pp. 405-414 (2005).

18. Zhang, G., Dillon, T.S., Cai, K.Y., Ma, J., and Lu, J. "Operation properties and $\delta$-equalities of complex fuzzy sets", International Journal of Approximate Reasoning, 50, pp. 1227-1249 (2009).

19. Aktas, H. and Cagman, N. "Soft sets and soft groups", Information Science, 177(13), pp. 2726-2735 (2007).

20. Aygunoglu, A. and Aygun, H. "Introduction to fuzzy soft groups", Computers and Mathematics with Applications, 58, pp. 1279-1286 (2009).

21. Acar, U., Koyuncu, F., and Tanay, B. "Soft sets and soft rings", Computers and Mathematics with Applications, 59(11), pp. 3458-3463 (2010).

22. Inan, E. and Ozturk, M.A. "Fuzzy soft rings and fuzzy soft ideals", Neural Computing and Applications, 21(1), pp. 1-8 (2011). 
23. Ghosh, J., Dinda, B., and Samanata, T.K. "Fuzzy soft rings and fuzzy soft ideals", International Journal of Pure and Applied Sciences and Technology, 2(2), pp. 66-74 (2011).

24. Zhu, K.Y. "Novel soft fuzzy rough rings (ideals) of rings and their application in decision making", Soft Computing - A Fusion of Foundations, Methodologies \& Applications, 23(9), pp. 3167-3189 (2019).

25. Vimala, J., Arockia Reeta, J., and Barathi, P. "Algebraic applications on I-fuzzy soft group", International Journal of Soft Computing, 12(5-6), pp. 322-328 (2017).

26. Zhan, J., Dudek, W.A., and Neggers, J. "A new soft union set: Characterizations of hemirings", International Journal of Machine Learning and Cybernetics, 8(2), pp. 525-535 (2017).

27. Bera, T. and Mahapatra, N.K. "On neutrosophic normal soft groups", International Journal of Applied and Computational Mathematics, 3(4), pp. 3047-3066 (2017).

28. Yamak, S., Kazanci, O., and Davvaz, B. "Soft hyperstructures", Computers and Mathematics with Applications, 62, pp. 797-803 (2011).

29. Leoreanu-Fotea, V., Feng, F., and Zhan, J. "Fuzzy soft hypergroups", International Journal of Computer Mathematics, 89(8), pp. 963-974 (2012).

30. Selvachandran, G. "Introduction to the theory of soft hyperrings and soft hyperring homomorphism", JP Journal of Algebra, Number Theory and Applications, 36(3), pp. 279-294 (2015).

31. Selvachandran, G. and Salleh, A.R. "Hypergroup theory applied to fuzzy soft sets", Global Journal of Pure and Applied Mathematics, 11(2), pp. 825-834 (2015).

32. Selvachandran, G. and Salleh, A.R. "Fuzzy soft hyperrings and fuzzy soft hyperideals", Global Journal of Pure and Applied Mathematics, 11(2), pp. 807-823 (2015).

33. Selvachandran, G. and Salleh, A.R. "Vague soft hypergroups and vague soft hypergroup homomorphism", Advances in Fuzzy Systems, 2014, pp. 1-10 (2014).

34. Selvachandran, G. and Salleh, A.R. "Algebraic hyperstructures of vague soft sets associated with hyperrings and hyperideals", The Scientific World Journal, 2015, pp. 1-12 (2015).

35. Khan, A., Farooq, M., and Davvaz, B. "On (M, N) intersectional soft interior hyperideals of ordered semihypergroups", Journal of Intelligent and Fuzzy Systems, 33(6), pp. 3895-3904 (2017).

36. Ma, X., Zhan, J., and Leoreanu-Fotea, V. "Rough soft hyperrings and corresponding decision making", Journal of Intelligent and Fuzzy Systems, 33(3), pp. 1479-1489 (2017).

37. Al-Husban, R., Salleh, A.R., and Ahmad, A.G. "Complex intuitionistic fuzzy subrings", AIP Conference Proceedings, 1784, pp. 1-7 (2016).
38. Al-Husban, R., Salleh, A.R., and Ahmad, A.G. "Complex intuitionistic fuzzy subrings", AIP Conference Proceedings, 1739, pp. 1-7 (2016).

39. Al-Husban, A. and Salleh, A.R. "Complex fuzzy group based on complex fuzzy space", Global Journal of Pure and Applied Mathematics, 12(2), pp. 1433-1450 (2016).

40. Alsarahead, M.O. and Ahmad, A.G. "Complex fuzzy subgroups", Applied Mathematical Sciences, 11(41), pp. 2011-2021 (2017).

41. Alsarahead, M.O. and Ahmad, A.G. "Complex fuzzy soft group", Journal of Quality Management and Analysis, 13(2), pp. 17-28 (2017).

42. Rosenfeld, A. "Fuzzy groups", Journal of Mathematical Analysis and Applications, 35, pp. 512-517 (1971).

\section{Biographies}

Shio Gai Quek is currently a Mathematics lecturer at UCSI College, Kuala Lumpur, Malaysia. He holds a PhD (Pure Mathematics) and BEdu (Mathematics) from Universiti Malaya (UM), Malaysia. He has 3 years of teaching experience and has thus far published several research articles in journals and conferences. His areas of interest include fuzzy algebraic and hyperalgebraic theory, fuzzy computing, soft computing and applications of fuzzy computing in engineering, and decision-making and robotics.

Ganeshsree Selvachandran is currently an Associate Professor in the Faculty of Business and Information Science at UCSI University, Kuala Lumpur, Malaysia. She holds a PhD (Pure Mathematics), an MSc (Pure Mathematics), and a BSc (Hons) (Mathematics) from Universiti Kebangsaan Malaysia (UKM), Malaysia, and has seven years of teaching experience. She has published close to 40 research articles in various international journals and also proceedings of national and international conferences. Her areas of interest include fuzzy computing, multicriteria decision making (MCDM), soft computing, neutrosophic set theory, applications of fuzzy and soft computing in engineering, economics and pattern recognition, as well as fuzzy algebra and hyperalgebra.

Bijan Davvaz is a Professor at Yazd University, Iran. He is a prolific researcher in the area of fuzzy mathematics and has published more than 550 journal articles thus far, with most of the articles published in high-ranking international journals. His areas of interest include fuzzy algebraic and hyperalgebraic theory, fuzzy computing, and the theoretical aspects of fuzzy mathematics.

Madhumangal Pal is a Professor of Mathematics at Vidyasagar University, Medinipore, India. He holds an MSc From Vidyasagar University and a PhD from the 
Indian Institute of Technology in Kharagpur, India and has more than 20 years of teaching experience. He has published more than 250 research articles in various national and international journals and is also the author of eight published books. His areas of research include algorithmic graph theory and fuzzy matrices, genetic algorithms and the design, and analysis of algorithms. 